August 18, $2020 \quad$ 0:33 WSPC/INSTRUCTION FILE $\quad$ tn`2018`a v15’prep

\title{
Seeing Relativity - III. Journeying within the Kerr metric toward the negative gravity region
}

\author{
Alain Riazuelo \\ Sorbonne Université, CNRS, UMR 7095, Institut dAstrophysique de Paris, 98 bis boulevard \\ Arago, 75014 Paris, France
}

\begin{abstract}
In this paper we study some features of the Kerr metric both from an analytic and a visual point of view by performing accurate raytracing in various situations. We focus on features that are unique to the maximal analytic extension of the Kerr metric as compared to that of the Schwarzschild or even the Reissner-Nordström one. A large number of new, yet underexplored phenomena appear, especially regarding the structure of bounded null geodesics and the aspect of the negative gravity regions whose visual characteristics are shown both from outside and inside it.

03.30.+p, 04.25.D-
\end{abstract}

Keywords: Relativistic ray tracing, black hole, Kerr metric, maximal analytic extension

\section{Introduction}

The Kerr metric was discover by R.P. Kerr in $1963^{1}$. It was soon realized that it describes the gravitational field of a spinning black hole of mass $M$ and angular momentum per unit of mass $a^{2}$. Those are the sole parameters that describe the metric but despise this, the exterior part of this metric (i.e., outside the horizon) corresponds to the exact description of real black holes, the number of which being several tens of millions in a Milky Way-type galaxy. The existence of black hole has been a long-standing debate which was progressively settled by the discovery of a growing number of (then) stellar "black hole candidates" whose status slowly shifted toward real black holes, the black hole population being later expanded by that of supermassive black holes.

The Kerr metric is therefore of utmost importance in astrophysics, and the aspect of a Kerr black hole has been the subjects of innumerable papers, e.g., 3|4|5|6|7. However most deal with the case of astrophysically realistic black holes which were born at some epoch in the past. Before the black hole formation, space-time structure (neglecting the expansion of the Universe) is that of a Minkowski space, and after black hole formation it becomes that of a spacetime with a future event horizon, separating the interior and the exterior of the black hole. But the Kerr metric is actually even richer if one considers its maximal analytic extension. Contrarily to astrophysical black holes, the maximal analytic extension of the Kerr metric describes eternal black holes, or more precisely eternal wormholes that connect several distinct universes and whose structure is much more complicated than that of 
the canonical Morris-Thorne wormholes 8 . Moreover there are several reasoons to doubt that such configurations are stable with respect to tiny ravitational perturbations ${ }^{9}$. It is therefore clear that such extension is highly unlikely to exist in our Universe, however it possesses a number of fascinating properties whose study is barely sketched in a only very small number of papers, such as Ref. 6. The aim of this paper is to explore more deeply some of the mathematical features that arise in this metric.

Indeed, the whole complexity of the Kerr metric does not appear immediately when one writes the metric in some coordinate system, since a metric is nothing more than a manifold which is locally isomorphic to $\mathbf{R}^{4}$, which is then locally isomorphic to Minkowski space. The Kerr metric is, on the other hand, a manifold with a much more complicated topological and causal structure than $\mathbf{R}^{4}$. Moreover, knowing the causal structure of a manifold does not suffice to describe the geodesic structures that exist in the manifold. We mean here that, for example, the fact that some part of spacetime are in an observer's past lightcone does not suffice for this region to be actually seen, that is, reached by null geodesics. But even when we know which regions can actually be seen, the knowledge of these geodesic structures is insufficient to give good insights on what this observer would actually see when travelling within this metric. Although this might seem provocative, we argue that one can hardly claim to understand what a metric is without having a good intuition of what it could look like from a visual point of view, and we think that the Kerr metric is a very good example of such claim. The aim of this paper is therefore to propose a numerical exploration of the maximal analytic extension of the Kerr metric from a visual point of view.

This paper is organized as follows. In $\$ 2$, we introduce the main mathematical quantities that are necessary in order to solve the geodesic equations. We use them in in $\$ 3$ to address a simple but unexplored case of the aspect of an astrophysical, realistic black seen from a very close distance. We then recall in \$4 the main features of the maximal analytic extension of the metric as well as the coordinate systems that are needed to deal with horizon crossings. An important feature of the maximal analytic extension is the existence of bounded geodesics the properties of which are studied in $\$ 5$. The visual consequences of them in some cases is shown in 86 Then, the most fascinating feature of the Kerr metric, that is an asymptotic region of negative gravity necessitates a new coordinate system and corresponding new geodesic equations, both of which are presented in 87 . With all this material we can now simulate in $\$ 8$ the journey of an observer all the way from a standard asymptotic region to a negative gravity region.

\section{Background material}

There exists a large number of review articles regarding the Kerr metric, e.g., $10 \mid 111213$. Although at large amount of common materiel is present in each of these references (and many others), each of them has its specificities. For example, Ref. 12 
addresses many mathematical aspects of the metric whereas Ref. 13 focuses more on geodesics. Still, these voluminous references do not exhaust such a vast subject, especially when one considers the maximal analytical extension of the metric, whose study is the aim of this paper.

Many coordinate systems can be used to study the Kerr metric, the most convenient one depending on the context. For example, the causal structure of the metric is more readily apparent when considering the Boyer-Lindquist coordinates, which are a generalization of the so-called Schwarzschild coordinates that are almost always used to introduce the Schwarzschild metric as well as the Reissner-Nordström one. However, if we need to handle horizon crossings, the Boyer-Lindquist coordinate system is inefficient. In the first part of this paper, we will not deal with horizon crossings, therefore we start by first using the Boyer-Lindquist coordinates.

\subsection{Boyer Lindquist coordinate system}

Using the (+- - ) metric signature convention, the non-zero metric coefficients in this coordinate system are written as

$$
\begin{aligned}
g_{t t} & =\frac{\Delta-a^{2} \mathrm{~s}^{2}}{\Sigma}=1-\frac{2 M r}{\Sigma}, \\
g_{t \varphi} & =\frac{2 M r a \mathrm{~s}^{2}}{\Sigma}, \\
g_{\varphi \varphi} & =-\mathrm{s}^{2} \frac{\left(r^{2}+a^{2}\right)^{2}-\Delta a^{2} \mathrm{~s}^{2}}{\Sigma}=-\mathrm{s}^{2}\left(r^{2}+a^{2}+\frac{2 M r a^{2} \mathrm{~s}^{2}}{\Sigma}\right), \\
g_{r r} & =-\frac{\Sigma}{\Delta}, \\
g_{\theta \theta} & =-\Sigma
\end{aligned}
$$

where we have defined

$$
\begin{aligned}
\mathrm{s} & \equiv \sin \theta, \\
\mathrm{c} & \equiv \cos \theta, \\
\Delta & \equiv r^{2}-2 M r+a^{2}, \\
\Sigma & \equiv r^{2}+a^{2} \cos ^{2} \theta .
\end{aligned}
$$

This metric is asymptotically flat but the $r$ coordinate becomes timelike when $\Delta=$ 0 , which translates into the fact that there are two horizons situated at

$$
r_{ \pm}=M \pm \sqrt{M^{2}-a^{2}} .
$$

The metric does not depend on the $t$ and $\varphi$ coordinates, which is a consequence of the fact that it is both stationary and axisymmetric. Consequently, for any geodesics 
there exists two obvious constants of motion, $E$ and $L_{z}$, defined as

$$
\begin{aligned}
E & \equiv \pi_{t}=g_{t a} \dot{x}^{a}=\left(1-\frac{2 M r}{\Sigma}\right) \dot{t}+\frac{2 M r a \mathrm{~s}^{2}}{\Sigma} \dot{\varphi} \\
L_{z} & \equiv-\pi_{\varphi}=-g_{\varphi a} \dot{x}^{a}=\left(r^{2}+a^{2}+\frac{2 M r a^{2} \mathrm{~s}^{2}}{\Sigma}\right) \mathrm{s}^{2} \dot{\varphi}-\frac{2 M r a \mathrm{~s}^{2}}{\Sigma} \dot{t}
\end{aligned}
$$

where a dot corresponds to derivation of the corresponding coordinate with respect to one of the geodesic affine parameter. Furthermore, for any geodesic, the norm $\kappa$,

$$
\kappa=g_{a b} \dot{x}^{a} \dot{x}^{b}
$$

is constant, and equal to either 1 for timelike trajectories or 0 for null geodesics. Finally, there exists a far less obvious constant of motion, the Carter constant, $C$ which in this coordinate system is defined as

$$
C=\pi_{\theta}^{2}+a^{2} c^{2} \kappa+\left(a E \mathrm{~s}-\frac{L_{z}}{\mathrm{~s}}\right)^{2} .
$$

\subsection{Equations of motion in Boyer-Lindquist coordinates}

The constants of motions $E$ and $L_{z}$ allow to simplify the geodesic equations which, for coordinates $t$ and $\varphi$, can then be written as first order equations: one can invert the equations defining $E$ and $L_{z}$ in order to obtain such closed forms for $\dot{t}$ and $\dot{\varphi}$. They are, respectively,

$$
\begin{aligned}
\Delta \dot{t} & =\left(r^{2}+a^{2}+\frac{2 M r a^{2} \mathrm{~s}^{2}}{\Sigma}\right) E-\frac{2 M r a}{\Sigma} L_{z}, \\
\Delta \dot{\varphi} & =\left(1-\frac{2 M r}{\Sigma}\right) \frac{L_{z}}{\mathrm{~s}^{2}}+\frac{2 M r a}{\Sigma} E .
\end{aligned}
$$

For the two other variables, the most compact form of the equations of motion reads

$$
\begin{aligned}
& \Sigma^{2} \dot{r}^{2}=R(r) \equiv\left(\left(r^{2}+a^{2}\right) E-a L_{z}\right)^{2}-\Delta\left(\kappa r^{2}+C\right), \\
& \Sigma^{2} \dot{\theta}^{2}=\Theta(\theta) \equiv C-a^{2} c^{2} \kappa-\left(a E \mathrm{~s}-\frac{L_{z}}{\mathrm{~s}}\right)^{2} .
\end{aligned}
$$

These two equations are not usable in practice as they do not allow to notice a possible change of sign in $\dot{r}$ or $\dot{\theta}$. In order to do so, one needs to consider the time derivative of these, which give

$$
\begin{aligned}
& \Sigma^{2} \ddot{r}=\frac{R^{\prime}}{2}-2 r \sum \dot{r}^{2}+2 \Sigma a^{2} \operatorname{cs} \dot{\theta} \dot{r}, \\
& \Sigma^{2} \ddot{\theta}=\frac{\Theta^{\prime}}{2}+2 a^{2} \Sigma \operatorname{cs} \dot{\theta}^{2}-2 r \sum \dot{r} \dot{\theta},
\end{aligned}
$$

where the primes denote a derivative with respect to $r$ (for $R$ ) and $\theta$ (for $\Theta$ ). These derivatives can be explicitly written as

$$
\begin{aligned}
\frac{R^{\prime}}{2} & =2 r E\left[\left(r^{2}+A^{2}\right) E-a L_{z}\right]-(r-M)\left(C+\kappa r^{2}\right)-\Delta \kappa r, \\
\frac{\mathrm{s}}{\mathrm{c}} \frac{\Theta^{\prime}}{2} & =a^{2} \mathrm{~s}^{2} \kappa-\left(a E \mathrm{~s}-\frac{L_{z}}{\mathrm{~s}}\right)\left(a E \mathrm{~s}+\frac{L_{z}}{\mathrm{~s}}\right) .
\end{aligned}
$$


Apart from the case of polar trajectory where the term $1 / \mathrm{s}$ may diverge, this set of equations is regular everywhere outside the outer horizon at $r=r_{+}$.

\section{Simulated views, 1st part}

The above equations are sufficient to address the issue of a Kerr black hole aspect for seen from the point of view of any observer outside the horizon. Almost all the literature dealing with raytracing in the Kerr metric focuses on the actual aspect of such black hole seen by a distant observer but almost none deals with the point of view of an observer much closer to the black hole. However, although this was not the initial aim of our work, we found that several interesting features arise when considering such observers, and we shall devote this Section to this issue. More specifically, whether or not the special case $a=M$ deserves attention in the study of the Kerr metric has been an open matter in the existing literature. For example, Ref. 12 argues that it does not. Our work tentatively brings some elements to this debate as we have found that several strange phenomena can arise in this case and were so far overlooked in previous studies 7 .

Going from the geodesic equations to full-fledged views of a celestial sphere is essentially independent of the metric one is considering, at least as long that it is asymptotically flat so that we can consider a static celestial sphere lying at infinity. The technical details have already been explained in a previous paper 14, so that we shall not give more details here.

\subsection{Shape of the black hole silhouette for an equatorial observer}

The case we have focused on is what we think to be the most extreme situation that can occur outside a rotating black hole horizon. It deals with an observer orbiting along a circular orbit around and close to an extremal Kerr black hole in the equatorial plane. The choice of an orbiting instead of a static observer is of course motivated by the fact that no static observer can lie within the ergosphere, i.e., close to the black hole. We therefore consider the case of an observer along a circular, equatorial geodesic and we impose the trajectory to be prograde as it allows smaller orbital radii. When the observer is at large distance, such a configuration is very close to that of a static observer since the Lorentz transform that allows to go from a moving to a static observer is close to identity, so that the resulting Doppler or aberration distortions are negligible. A well-known result regarding the shape of an extremal black hole silhouette it that from the point of view of a distant, static observer, it looks like a disk with a flattened side. Assuming that the black hole is spinning counterclockwise from the point of view of an equatorial observer, the flattened side is on the left. This asymmetry can more or less intuitively understood by the fact that there exist equatorial null geodesics, whose minimal coordinate radii are very different whether they and prograde or retrograde. These radii are given by $r_{\mathrm{p}}$ and $r_{\mathrm{i}}$ of Eq. (79) and respectively tend to $M$ and $4 M$ when $a \rightarrow M$. Consequently, null equatorial geodesics performing a close flyby near the black hole 
can also reach values close to $M$ or $4 M$ at their closest approach. Consequently the "prograde radius" of the black hole (i.e., the side on which flyby geodesics reaching the observer travel in the same direction as prograde circular geodesics ones) is much smaller than the "retrograde radius". The net result of these features is that the black hole silhouette is asymmetric, it is flattened along the prograde side. However this silhouette is still convex but there is no obvious reason that it should be the case, nor that it should be the case for an observer standing at a finite distance. We investigate this question in the next paragraphs.

In order to simulate images, we need to decide what the celestial sphere looks like. We (rather arbitrarily) chose a celestial sphere corresponding to the sky seen from Earth we are familiar with. When computing a distorted celestial sphere, a different treatment must be applied of the celestial sphere itself and the pointlike images of the stars 14 . Since we are more interested in the distortions of the images, we decided to abruptly remove all the stars from the celestial sphere, which is taken as infrared sky as seen from the 2MASS survey 15 .

The images are now computed at a resolution of $3600 \times 3600$ pixels. In order to reduce the number of views and to allow seeing every direction, pictures have been computed in fish-eye format that is adapted to digital planetarium, under the so-called DomeMaster format: the disk that fits inside the images correspond to exactly $2 \pi$ steradians (i.e., a half sphere). Aberration and Doppler effect are taken into account, although imperfectly for the latter, since only a bolometric correction is applied to pixel intensity (intensity changes, but not hue). We shall show below and comment the views for decreasing values of the orbital radius $r$, which can be as small as $M$.

\section{2. $r=6 M$}

We first show in Fig. 1 the aspect of the black hole silhouette at moderate distance, i.e., coordinate distance $r=6 M$, which is the innermost stable circular orbit in the Schwarzschild metric. The silhouette shape is qualitatively similar to that at infinite distance, although it is more clearly elongated along the polar direction than along the equatorial one. (This was already the case at large distance, but far less obvious to notice by eye.) Another interesting feature is that although we expect to have an infinite number of multiple images of the celestial sphere around the black hole silhouette, these images are easier to see on the prograde size in the sense that their angular separation is larger. In the rather featureless celestial sphere image we chose, the multiple images are those of the Milky Way disk.

\section{3. $r=1.5 M$}

Fig. 2 shows what happens at $r=1.5 M$. Below $r=2 M$, the observer lies in the ergoregion and the shape of the black hole silhouette significantly changes (although in a regular and continuous manner). It is no longer convex, both along the prograde 


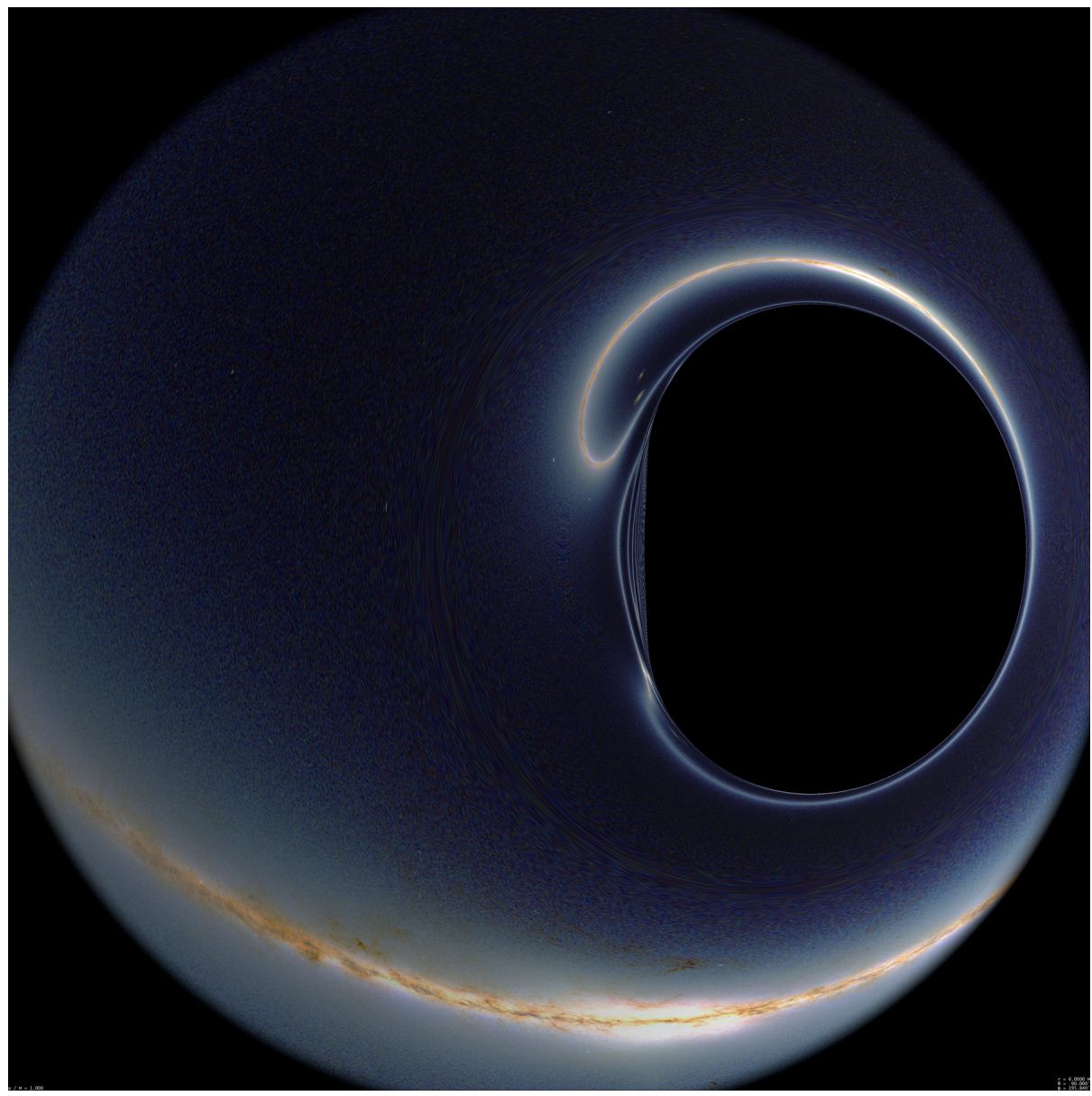

Fig. 1. $r=6 M$, overall black hole silhouette.

side and, more unexpectedly, along the retrograde side, taking overall a peanutlike shape. Another interesting feature is that, just as in the previous figure, the multiple images in the prograde side are separated by a much larger angle that on the retrograde size.

Note also that determining the direction toward which the observer is heading is not easy from now on since we are beyond the static limit. The concept of direction makes sense in a static metric: as compared to a static observer of four-velocity $u_{\text {stat }}=\partial / \partial t$, another observer's arbitrary four-velocity $v^{a}$ can always be written $v^{a}=\gamma\left(u_{\text {stat }}^{a}+\beta n^{a}\right)$, where $n^{a}$ is a unit spacelike vector orthogonal to $u_{\text {stat }}^{a}$ and $\gamma=1 / \sqrt{1-\beta^{2}}$ is the Lorentz factor $v_{a} u_{\text {stat }}^{a}$. In such a configuration, one can state that the second observer is heading toward direction $n^{a}$, but the same procedure can no longer be implemented within the ergoregion (and, of course, within the horizon). From now on, the choice of the "front" and "rear" direction is rather arbitrary. We 
shall adjust our images so that the center of the "front" image corresponds to the most blueshifted part of the celestial sphere, keeping in mind that even outside the ergoregion (and even in the Schwarzschild case) this does not correspond to the direction of motion as we just have defined it.
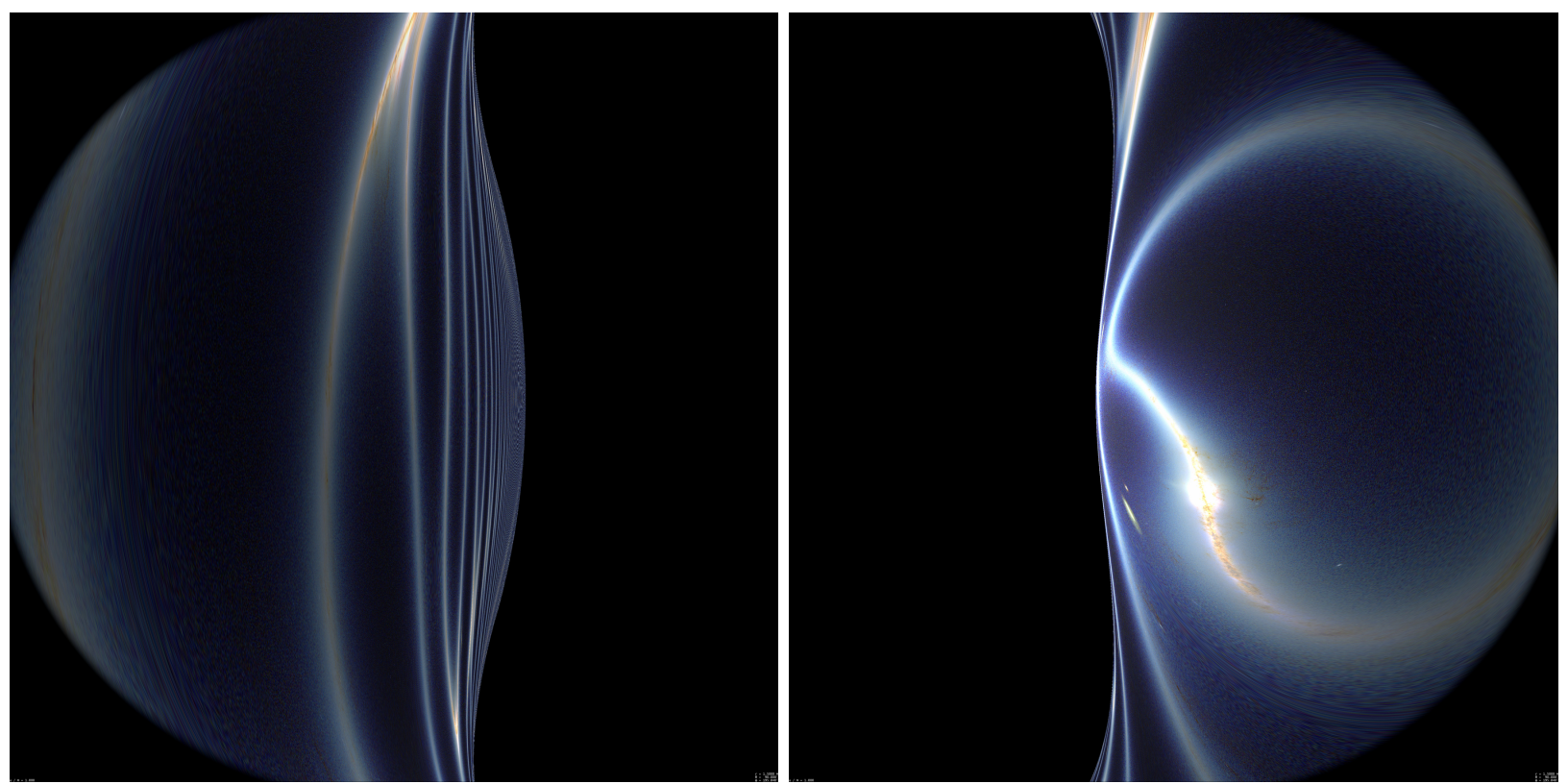

Fig. 2. $r=1.5 M$, rear view (left) and front view (right).

\subsection{Going deeper in the ergosphere}

Going deeper in the ergoregion, several effects arise.

Firstly the silhouette is still peanut-shaped but in an increasingly asymmetric way. The concave part along the prograde side is quite close to a circular arc, whereas on the retrograde side it becomes increasingly pinched.

Secondly there are more multiple images that are visible on the prograde side than on the retrograde one but their relative positions are quite different. On the prograde side, their separation decreases as one gets closer to the black hole silhouette. This is indeed what one could expect given what happens in the Schwarzschild case, however the big difference here is that the separation between two multiple images decreases rather slowly (as opposed to exponentially in the Schwarzschild case, see, e.g., Ref. 12).

Thirdly, on the retrograde side, the multiple images get organized along a very regular pattern. For what we use as an image here (the Milky Way disk), they appear as circles (or water droplets) which are tangent at the the "pinch" of the 
black hole silhouette.

Figure 3 illustrates these three effects as they are see at $r=1.05 \mathrm{M}$
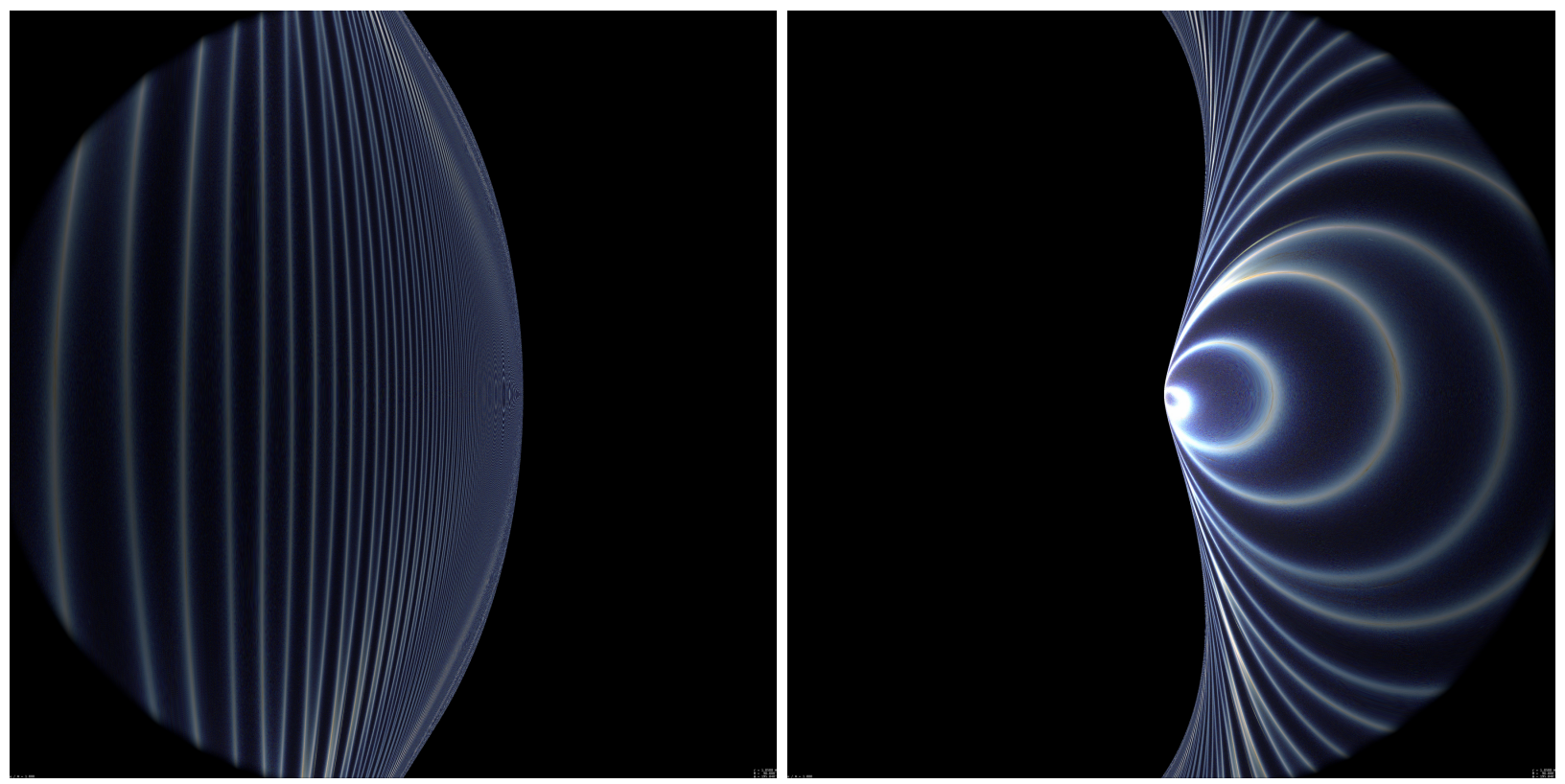

Fig. 3. $r=1.05 M$, rear view (left) and front view (right).

\subsection{Skimming over the horizon}

In the Schwarzschild metric, the aspect of the black hole does not drastically change when a freely-falling observer approaches the horizon, but this is not what happens here in the Kerr metric, although in a slightly different context since we are considering a series of orbiting observer along similar circular orbits. The view at $r=1.005 M$ (Fig. 4 ) is qualitatively close to that of Fig. 3 except that the patterns we mentioned on the retrograde side are even more regular, with something like ten times more images on the retrograde side. This may suggest that the spacing between the images scales as $M /(r-M)$, but this is merely a conjecture. The number of images on the prograde side is not so high that one is limited by the screen resolution.

The visual limit of what can be obtained with $1800 \times 1000$ hemispheric view is reached around $r=1.001 M$ on the retrograde side(see Fig. 5). For the prograde side, the limit depends on where on is looking since the spacing between multiple image is less regular, but the limit is obtained for larger $r$ (see Fig. 4).

If we improve the picture resolution, so does the limit at which one can see details (Fig. 6). However a larger number of technical difficulties arise at some 

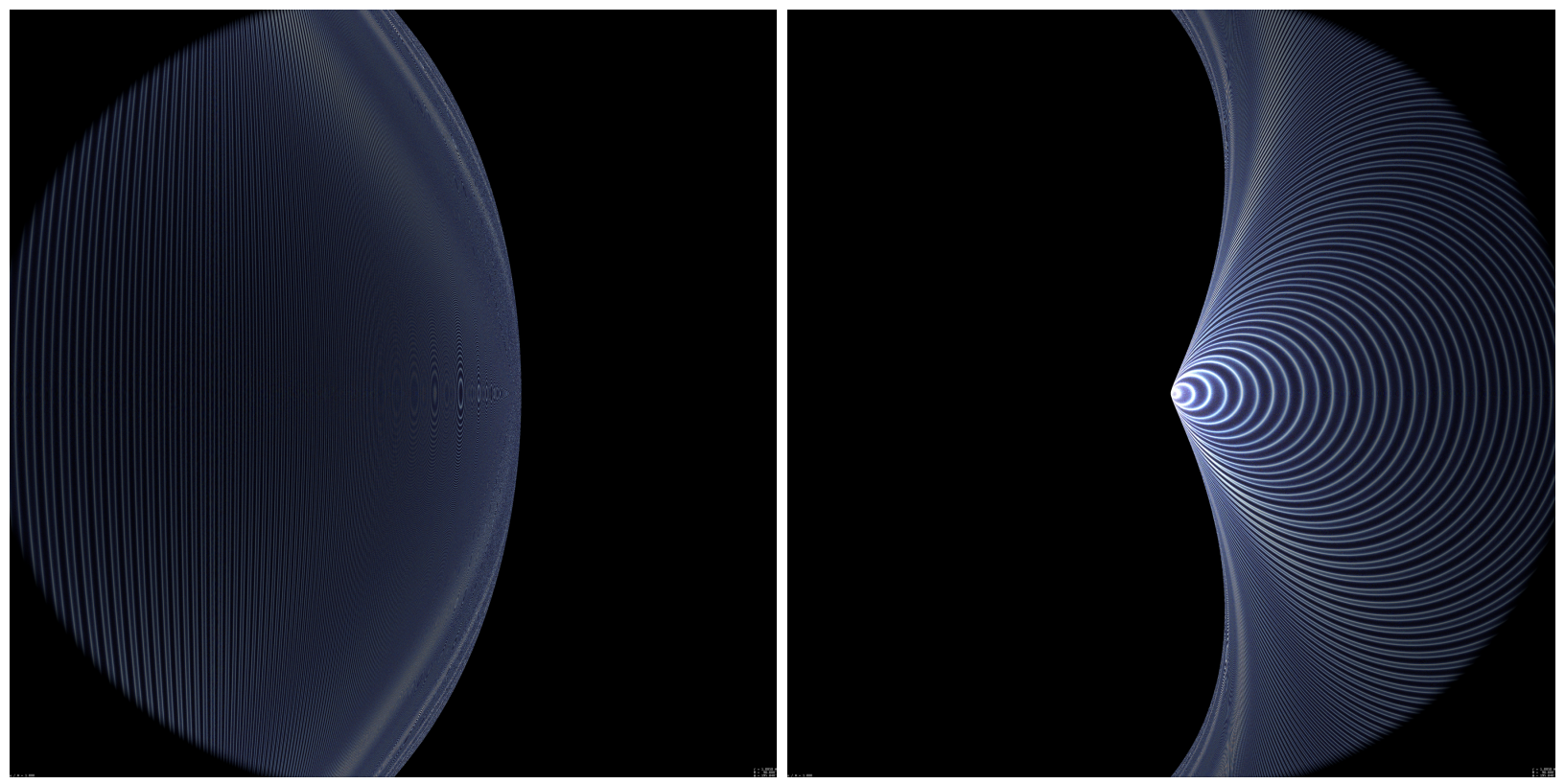

Fig. 4. $\quad r=1.005 M$, rear view (left) and front view (right). The Moiré-like effects on the left image are not physical features but instead limitation due to the insufficient resolution of the computer-generated view.

point which all come from the combination of the observer's position and velocity. When $r$ is very close to the horizon, there exists some risk that the Boyer Lindquist coordinate system becomes inappropriate. This is true, unfortunately, this is also the case for the Kerr-Schild coordinates. Indeed, one might think that since the observer is looking in all directions (since we compute two hemispheric views), many of the geodesics make a significant angle with respect to the horizon. However, the observer we are considering is very specific here. As compared with a freely falling observer with zero velocity and angular momentum, it is necessary to perform a huge Lorentz boost to pass from this first fiducial observer's reference frame to that of the observer that actually sees the celestial sphere. Because of this most of the directions of the circularly orbiting observer sees correspond to a very thin bundle of directions from the point of view of the freely-falling observer, and this bundle of direction correspond to photons that reach the observer with a very low $\dot{r}$. For this reason, the coordinate change from Boyer-Lindquist to Kerr-Schild coordinate does not affect the $t, \varphi$ coordinates: we have by construction $\dot{T} \sim \dot{t}$ and $\dot{\tilde{\varphi}} \sim \dot{\varphi}$, which are both very large. Such situation is numerically very unstable and has a very bad impact on the computational time for each geodesic of the screen (but a very small number of these). Consequently, the CPU time increases very rapidly when the circularly observer's orbital radius gets closer and closer to the horizon, $r_{\text {hor }}=M$. 

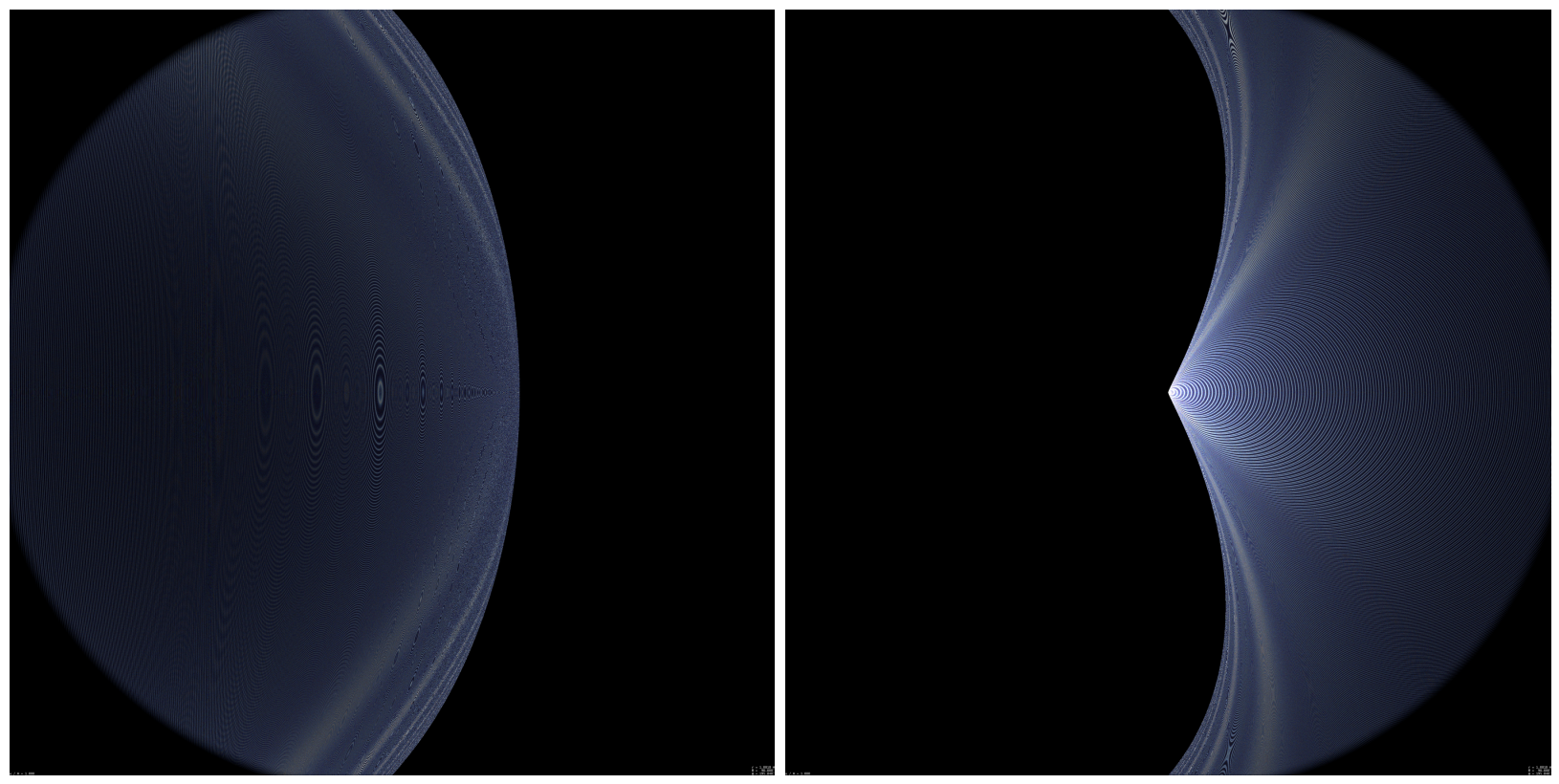

Fig. 5. $\quad r=1.001 M$, rear view (left) and front view (right). At this resolution, only the front view remains (barely) accurate.

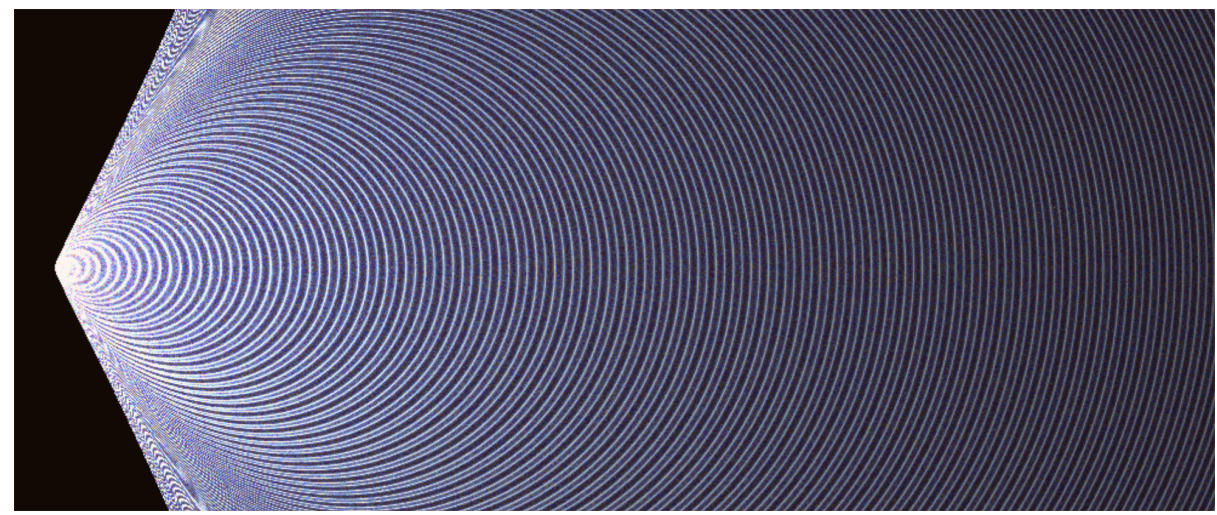

Fig. 6. Snapshot of a 3600 pixel wide hemispheric view at $r=1.0005 M$. This view covers a $50^{\circ}$ wide field, i.e. with an effective screen resolution of 3 arcmin, not much worse than human eye resolution.

We stop here our numerical investigation of the exterior aspect of the Kerr metric. Several of the features we discovered here, most notably the regular image spacing on the retrograde side, as well as the pinched aspect of the black hole silhouette from this side as well, obviously deserve a rigorous derivation but we leave this for a future work. 


\section{Classifying geodesics}

The ultimate aim of this paper is to visualize the maximal analytic extension of the Kerr metric. Wherever the observer lies, it will intersect geodesics coming from some past horizons and traveling from different asymptotic regions than that it originates from. We therefore first need to address which regions can be seen as a function of the region the observer lies in.

\subsection{Reminder on the maximal analytic extension of the Kerr metric}

The Carter-Penrose diagram of the Kerr metric has been described decades ago by several authors, see, e.g., 17 and $\stackrel{16}{16}$ for the historical reference. We shall first consider an observer lying in some asymptotic region, i.e., a region of positive $r$ and outside the outer horizon (that is, $r>r_{+}$). This region is bounded by the black hole outer horizon at $r=r_{+}$. In a causal diagram, it is represented by a diamondshaped region whose edges are inclined at $\pm 45^{\circ}$ with respect to horizontal/vertical directions. The null ingoing and outgoing directions ( $E^{a}$ and $S^{a}$, respectively) are defined by the null vectors such that $\kappa=C=0, L_{z}=a \mathrm{~s}^{2} E$ whose components are therefore, up to some arbitrary constant,

$$
S^{a}=\left(\begin{array}{c}
\frac{r^{2}+a^{2}}{\Delta} \\
1 \\
0 \\
\frac{a}{\Delta}
\end{array}\right), E^{a}=\left(\begin{array}{c}
\frac{r^{2}+a^{2}}{\Delta} \\
-1 \\
0 \\
\frac{a}{\Delta}
\end{array}\right)
$$

Then, switching from $r$ to the tortoise coordinate $r^{*}$ such that $\mathrm{d} r^{*}=\frac{r^{2}+a^{2}}{\Delta} \mathrm{d} r$, the hypersurface spanned by $E^{a}$ and $S^{a}$ is causally equivalent to that of a twodimensional Minkowski spacetime in each intervals of $r$ where $r^{*}$ is regular, that is in the three intervals $] r_{+}, \infty[,] r_{-}, r_{+}[]-,\infty, r_{-}[$. Consequently, in a Carter-Penrose diagram, all these regions are diamond-shaped. Considering the region $r>r_{+}$, the lower right edge of its patch corresponds to past null infinity $(t-r \rightarrow-\infty$, or, equivalently, $\left.t-r^{*} \rightarrow-\infty\right)$, the upper right edge to future null infinity $(t+r \rightarrow \infty)$. The upper left edge corresponds to the future outer horizon $\left(r=r_{+}, t \rightarrow \infty\right.$, or $\left.t-r^{*} \rightarrow \infty\right)$, and the lower left edge to the past outer horizon $\left(r=r_{+}, t \rightarrow-\infty\right)$. In this region, $\Delta$ is always positive, so that $r$ is a spacelike coordinate. Conversely, $t$ is almost everywhere a future oriented timelike coordinate, except near the black hole when $g_{t t} \propto \Delta-a^{2} \mathrm{~s}^{2}$ can become negative for sufficiently small $r$ (unless $\theta=0, \pi$ ), a configuration which forms the outer ergoregion. The fact that $t$ is both timelike and future-oriented means that null geodesics coming from or heading toward null infinity have a positive $E=\pi_{t}$. Geodesics with negative $E$ can exist in this region, however they cannot go further (in term of $r$ coordinate) than the limit of the outer ergoregion. We shall label this region, including the ergoregion, 1. 
The next patch of the Carter-Penrose diagram is the inter horizon region, which we call region 2 . There, $\Delta<0$, so that $r$ is a timelike coordinate. Since this coordinate can only decrease when one crosses the outer horizon, $r$ is past-oriented. Region 2 is adjacent to region 1 through its lower right edge. Its lower left edge is adjacent to another asymptotic region which has the same structure as region 1 , except that $t$ is past-oriented. We shall label it region 3 . This second asymptotic region already exists in both the Schwarzschild and Reissner-Nordstrm metric, and so does region 2 except that it is bounded from above by the singularity in the Schwarzschild cas@

Region 2 upper edges correspond to $r=r_{-}$and $t \rightarrow \pm \infty$ and connect to two new patches. These both possess an inner ergoregion and both share similar properties, except that in one patch, $t$ is future-oriented outside the ergoregion, whereas it is past-oriented in the other. Since radial null geodesics from region 1 travel from right to left in the Carter-Penrose diagram, it is the left $r<r_{-}$patch whose $t$ coordinate is future oriented out of its ergoregion. We shall label it region 5, its right counterpart being region 6 .

Each regions 5 and 6 , which are diamond-shaped, can further be split into two parts, one with $r>0$, and one with $r<0$, which is another asymptotic region, which we shall call 7 and 8 , respectively. Since $t$ is future oriented in region 7 , geodesics traveling there have positive $E$, whereas all geodesics of region 8 coming from past null infinity have a negative $E$. Apart from the $r<0$ part, regions 5 and 6 also exist (without ergoregion) in the Reissner-Nordström metric, where they are bounded from one side by the then uncrossable pointlike singularity at $r=0$.

Regions 5 and 6 are bounded from above by a second inter-horizon region which has the same properties as region 3, except that $r$ is now future oriented. We shall label this region 12. The two upper edges of region 12 correspond to $r=r_{+}, t \rightarrow \pm \infty$ then connect to two new asymptotic region which we shall label 9 (to the right) and 11 (to the left). By a similar reasoning as above, we know that outside their respective ergoregions, $t$ is future-oriented in region 9 and past-oriented in region 11.

The full analytic extension of the Kerr metric is then an infinite tower of six diamond-shaped patches whose labels will be deduced from those of the neighboring block by adding or subtracting 8 . The labels we choose are summarized in Figure 7 It is also to be noted that dubbing this diagram as a causal one is slightly misleading as a small patch of the $r<0$ region allows for causality violations 18 all the way to the neighboring $0<r<r_{-}$region by using some specific displacement along the $\varphi$ coordinate. This does not affect the discussion that follows nor the appearance of asymptotic regions seen from there, at least as long as we assume that the past null infinities of those regions can be considered as static, which is what we shall do there.

aThere does not seem to be any consensus on how to label these patches. For example, Ref. 17 use Roman I for both our regions 1 and 3, and II for our 2 and 4 (see below), whereas as Ref. 12 use I, II, III, I', II', III' for our 1, 2, 5, 3, 4, $6 \bmod 8$, respectively. 


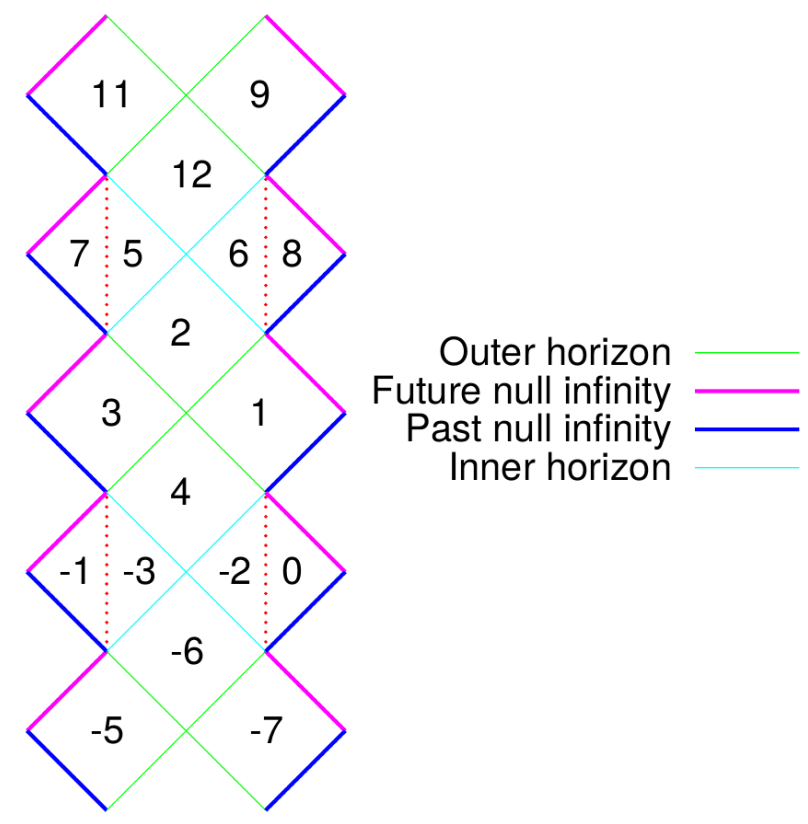

Fig. 7. Carter-Penrose diagram showing the causal structure of the full analytical extension of the Kerr metric. A bit more than two blocs of the diagram is shown here, although the full diagram is made of an infinite series of such blocks. Normal asymptotic regions (i.e., 1,3 , and $1+8 k, 3+8 k$ for any integer $k$ ) are bounded on one side by the outer horizon and from the other side by past and future null infinity. Other asymptotic region with negative $r$ exist as well, where wormhole gravity is negative. Those are regions $7+8 k, 8 k$. Normal asymptotic regions are partially filled with the outer ergoregion, whereas inner regions (labeled $5+8 k, 6+8 k$ ), which are bounded by $r=r_{-}$and $r=0$ also contain an inner ergoregion.

\subsection{Dealing with horizon crossings by using Kerr-Schild coordinates}

When leaving region 1 to enter region $2, t$ goes to infinity and is therefore not a wellbehaved coordinate. It has to be replaced by another coordinate, $T$ (together with $\varphi$ being replaced by $\tilde{\varphi}$ ), which are the so-called Kerr-Schild coordinates. Starting from the Boyer-Lindquist coordinates, they are defined as

$$
\begin{aligned}
\dot{T} & \equiv \dot{t}+\epsilon \frac{2 M r}{\Delta} \dot{r}, \\
\dot{\varphi} & \equiv \dot{\varphi}+\epsilon \frac{a}{\Delta} \dot{r},
\end{aligned}
$$

where $\epsilon$ is defined up to its sign, i.e., $\epsilon= \pm 1$. The appropriate choice of sign depends on the horizon crossing one is interested in, which we shall study in a few paragraphs. The important point is that both values of $\epsilon$ are mandatory, depending 
on the geodesics and the horizon crossings we have to deal with. Therefore, there are, in practice, two distinct Kerr-Schild coordinate systems, $\left(T_{-}, \tilde{\varphi}_{-}\right)$and $\left(T_{+}, \tilde{\varphi}_{+}\right)$ depending on the value of $\epsilon$. We shall however drop tho,+- subscript as long as it does not induce any confusion on which coordinate system is being used. Before dealing with the choice of $\epsilon$, we shall give the new version of the equation of motion in the Kerr-Schild coordinates.

Firstly, regarding the variable themselves, one can express $T$ and $\tilde{\varphi}$ as a function of $t, r$, and $\varphi, r$, respectively. The transformation depends on the value of $M^{2}-a^{2}$. When this quantity is positive (i.e., the metric describes a black hole), one has

$$
\begin{aligned}
& T=t+\frac{\epsilon M^{2}}{\sqrt{M^{2}-a^{2}}} \ln \left|\frac{r-r_{+}}{r-r_{-}}\right|+\epsilon M \ln \left|\frac{\Delta}{M^{2}}\right|, \\
& \tilde{\varphi}=\varphi+\frac{\epsilon a}{2 \sqrt{M^{2}-a^{2}}} \ln \left|\frac{r-r_{+}}{r-r_{-}}\right| .
\end{aligned}
$$

In the extremal, $a=M$ case, one has

$$
\begin{aligned}
& T=t+2 \epsilon M \ln \left|\frac{r-M}{M}\right|-2 \epsilon \frac{M^{2}}{r-M}, \\
& \tilde{\varphi}=\varphi-\frac{\epsilon M}{r-M} .
\end{aligned}
$$

Secondly, in both cases, the metric in term of the Kerr-Schild coordinates can be rewritten in the coordinate singularity free form,

$$
\begin{aligned}
\mathrm{d} s^{2}= & \mathrm{d} T^{2}-\mathrm{d} r^{2}-\Sigma \mathrm{d} \theta^{2}+2 \epsilon a \mathrm{~s}^{2} \mathrm{~d} r \mathrm{~d} \tilde{\varphi}-\left(r^{2}+a^{2}\right) \mathrm{s}^{2} \mathrm{~d} \tilde{\varphi}^{2} \\
& -\frac{2 M r}{\Sigma}\left(\mathrm{d} T+\epsilon \mathrm{d} r-a \mathrm{~s}^{2} \mathrm{~d} \tilde{\varphi}\right)^{2}
\end{aligned}
$$

Although it is not obvious, the first part of this expression (the one without $M$ ) corresponds to a Minkowski metric expressed in spheroidal coordinates, so that the whole metric is in fact of the form $g_{a b}=\eta_{a b}-A l_{a} l_{b}$, where $\eta_{a b}$ is a Minkowski metric and $l_{a}=\left(1, \epsilon, 0,-a \mathrm{~s}^{2}\right)$ is a null vector ${ }^{b}$, either with respect to $g_{a b}$ or $\eta_{a b}$, and $A$ is a function given by the simple form $A=2 M r / \Sigma$. A very useful quantity that we shall use afterward is $W$, defined as

$$
W \equiv l_{a} u^{a}=\dot{T}+\epsilon \dot{r}-a \mathrm{~s}^{2} \dot{\tilde{\varphi}}
$$

where $u^{a}$ is the four-velocity/four momentum of the geodesic we are considering. Using this $W$, a large number of expressions can be rewritten by getting rid of either $\dot{T}$ or $\dot{\tilde{\varphi}}$. In particular, the constants of motions $E$ and $L_{z}$ defined in Eqns. 1112

\footnotetext{
${ }^{\mathrm{b}}$ This null vector is the contravariant form of either $E^{a}$ or $S^{a}$ of Eq. 23 .
} 
can be rewritten

$$
\begin{aligned}
\Sigma E & =\left(\Delta-a^{2} \mathrm{~s}^{2}\right) \dot{T}+2 M r a \mathrm{~s}^{2} \dot{\tilde{\varphi}}-\varepsilon 2 M r \dot{r} \\
& =\Sigma \dot{T}-2 M r W \\
& =(\Sigma-2 M r) W-\Sigma \epsilon \dot{r}+\Sigma a \mathrm{~s}^{2} \dot{\tilde{\varphi}} \\
\Sigma \frac{L_{z}}{\mathrm{~s}^{2}} & =\left(\Sigma\left(r^{2}+a^{2}\right)+2 M r a^{2} \mathrm{~s}^{2}\right) \dot{\varphi}-2 M r a \dot{T}-\epsilon(2 M r+\Sigma) a \dot{r} \\
& =\left(\left(r^{2}+a^{2}\right)^{2}-\Delta a^{2} \mathrm{~s}^{2}\right) \dot{\varphi}-2 M r a \dot{T}-\epsilon(2 M r+\Sigma) a \dot{r} \\
& =\Sigma\left(r^{2}+a^{2}\right) \dot{\tilde{\varphi}}-2 M r a W-\epsilon a \Sigma \dot{r} .
\end{aligned}
$$

Several combinations of those expressions are useful. In particular,

$$
\begin{aligned}
a E-\frac{L_{z}}{\mathrm{~s}^{2}} & =a \dot{T}-\left(r^{2}+a^{2}\right) \dot{\tilde{\varphi}}+\epsilon a \dot{r} \\
& =a W-\Sigma \dot{\tilde{\varphi}} \\
\left(r^{2}+a^{2}\right) E-a L_{z} & =\Delta W-\epsilon \Sigma \dot{r}, \\
\Sigma E-\Delta W & =-a^{2} \mathrm{~s}^{2} W-\Sigma \epsilon \dot{r}+\Sigma a \mathrm{~s}^{2} \dot{\tilde{\varphi}} .
\end{aligned}
$$

Thirdly, Eqns. 15 16 are rewritten as:

$$
\begin{aligned}
& \Delta \dot{T}=\left(r^{2}+a^{2}+\frac{2 M r a^{2} s^{2}}{\Sigma}\right) E-\frac{2 M r a}{\Sigma} L_{z}+2 M r \epsilon \dot{r}, \\
& \Delta \dot{\tilde{\varphi}}=\left(1-\frac{2 M r}{\Sigma}\right) \frac{L_{z}}{\mathrm{~s}^{2}}+\frac{2 M r a}{\Sigma} E+\epsilon a \dot{r} .
\end{aligned}
$$

Because these equations have a $\Delta$ factor in their left-hand side, there is nothing that guarantees that the Kerr-Schild coordinates are indeed regular at horizon crossing, but this can actually be the case.

\subsection{Shortcut equations of motion in Kerr-Schild coordinates}

Indeed this issue can be overcome by noting that Eq. (17) can, rather obviously, be rewritten as

$$
\left[\left(r^{2}+a^{2}\right) E-a L_{z}-\epsilon \Sigma \dot{r}\right]\left[\left(r^{2}+a^{2}\right) E-a L_{z}+\epsilon \Sigma \dot{r}\right]=\Delta\left(C+\kappa r^{2}\right) .
$$

This ensures that one of the terms in the left-hand side of this equation is zero at horizon crossing. Eq. (35) shows that it is the second one that cancels. Indeed, this equation can be rewritten

$$
\left(r^{2}+a^{2}\right) E-a L_{z}+\epsilon \Sigma \dot{r}=\Delta W .
$$

Combining the last two equations, the $\Delta$ 's cancel out and one can obtain an interesting closed form for $W$ :

$$
W=\frac{C+\kappa r^{2}}{\left(r^{2}+a^{2}\right) E-a L_{z}-\epsilon \Sigma \dot{r}} .
$$


At horizon crossing, neither $\Sigma$ nor $\dot{r}$ can be 0 , therefore, there exists one choice of $\epsilon$ for which the denominator of the above equation is not 0 and hence this equation is regular at horizon crossing. Then, using the second version of Eqns. (32 34), one obtains a regular, "shortcut" version of the equations of motion for $T$ and $\tilde{\varphi}$ :

$$
\begin{aligned}
& \dot{T}=E+\frac{2 M r}{\Sigma} \frac{C+\kappa r^{2}}{\left(r^{2}+a^{2}\right) E-a L_{z}-\epsilon \Sigma \dot{r}}, \\
& \dot{\tilde{\varphi}}=\frac{1}{\Sigma}\left(\frac{L_{z}}{\mathrm{~s}^{2}}-a E\right)+\frac{a}{\Sigma} \frac{C+\kappa r^{2}}{\left(r^{2}+a^{2}\right) E-a L_{z}-\epsilon \Sigma \dot{r}} .
\end{aligned}
$$

This extends a similar version found for the Schwarzschild for the $T$ coordinate only. metric 19 .

Equation 411 immediately allows to understand which is the proper choice of $\epsilon$ at horizon crossing. Firstly, from the value of $r$ and $\dot{r}$ at some given time, it is possible to know which is the next horizon crossing, if any: (i) if $r<r_{-}$, then the next horizon crossing (if any) occurs at $r_{-}$, such as $\dot{r}_{\text {hor }}>0$, (ii) if $r>r_{+}$, the next horizon crossing (in case it occurs) is at $r=r_{+}$and will occur as $\dot{r}_{\text {hor }}<0$, and (iii) if $r_{-}<r<r_{+}$, there is certainly a horizon crossing, which occurs at $r_{+}$if $\dot{r}>0$ and at $r_{-}$otherwise, and the sign of $\dot{r}$ will then be the same as it is at the current time. Secondly, knowing the value $r_{\text {hor }}$ of the next horizon to be crossed, we can compute $\left(r_{\text {hor }}^{2}+a^{2}\right) E-a L_{z}$ and, most importantly its sign. Thirdly, we choose $\epsilon$ so as to ensure that both terms of the denominator of Eq. 411) are of same sign:

$$
\epsilon=-\operatorname{sgn}\left(\left.\dot{r}\right|_{r_{\text {hor }}}\right) \operatorname{sgn}\left[\left(r_{\text {hor }}^{2}+a^{2}\right) E-a L_{z}\right] .
$$

The same reasoning if of course valid if we integrate the geodesic backward in time. The only difference is then that the sign of $\dot{r}_{\text {hor }}$ has to be flipped in cases (i) and (iii).

\subsection{Second order equation of motion for $T$ and $\tilde{\varphi}$}

It is of course not mandatory to resort to using a first order equations such as the "shortcut" Eqns. 42 43 for the evolution of $T, \tilde{\varphi}$. It is also possible to find a second order differential equation for these variables by computing the proper time/affine parameter derivative of Eqns. (37/38) and by grouping terms so that everything is proportional to $\Delta$, which can be be canceled out. This is actually more conveniently done by considering the derivative of $\Delta W$ starting from Eq. (35). This derivative possesses a term proportional to $\ddot{r}$ in the right-hand side, which, starting from Eq. (21), must be recast into the largest possible amount of terms that are proportional to $\Delta$. After a few manipulations, we obtain

$$
\frac{R^{\prime}}{2}=\Delta X+\epsilon \Sigma \dot{r}(-2 r E+2 W(r-M)),
$$

where we have defined $X$ as

$$
X=2 r E W+(M-r) W^{2}-\kappa r .
$$


In order to obtain this rather compact form in Eq. 45, we made use of the following equality:

$$
C+\kappa r^{2}=W(\Delta W-2 \epsilon \Sigma \dot{r}),
$$

which is easily deduced from Eqns. (35) and (17).

Once all this is set, proper time/affine parameter derivative of Eq. 35 takes the very simple form

$$
\Sigma \dot{W}=\epsilon X
$$

from which we obtain

$$
\begin{aligned}
& \Sigma^{2} \ddot{\tilde{\varphi}}=-2 \dot{\theta} \Sigma \frac{\mathrm{c}}{\mathrm{S}} \frac{L_{z}}{\mathrm{~s}^{2}}+\epsilon a X-\Sigma \dot{\tilde{\varphi}} \dot{\Sigma} \\
& \Sigma^{2} \ddot{T}=2 M \dot{r} \Sigma W+2 M r \epsilon X-2 M r W \dot{\Sigma} .
\end{aligned}
$$

Although the three previous equations look regular regardless the value of $\epsilon$, this is not the case. The reason comes from the presence of the term proportional to $W^{2}$ in Eq. (48) through variable $X$ (see Eq. (46)). If we forget about all the other terms and assume that we are close to horizon crossing, so that $r$ and $\Sigma$ can be considered as constant, the (very) simplified form of Eq. (48) is

$$
\dot{W} \sim \epsilon \frac{M-r_{\text {hor }}}{\Sigma_{\text {hor }}} W^{2},
$$

whose solution is of the form, after defining $\alpha:=\left(M-r_{\text {hor }}\right) / \Sigma_{\text {hor }}$,

$$
\frac{W_{0}}{W}=1-\epsilon \alpha W_{0}\left(p-p_{0}\right),
$$

where the subscript 0 denotes the value at the start of integration (i.e., a short time before horizon crossing) and $p$ is the geodesic affine parameter. This equation has some chance to remain regular (i.e. $W$ will not blow up) only if $1 / W$ does not go to 0 , i.e. if $\epsilon$ has an opposite sign to that of $\alpha W_{0}$, which indeed explains why only one choice of $\epsilon$ can be valid at horizon crossing.

\subsection{Values of $\epsilon$ in the causal diagram}

We now want to address which values of $\epsilon$ are necessary for the Kerr-Schild coordinates to be suitable for each horizon crossing of the causal diagram. For this purpose, it suffices to know which value of $\epsilon$ has to be chosen for one geodesic.

Starting from the definition of the radial null vectors $E^{a}$ and $S^{a}$ (Eq. 23), it is clear that the components of $E^{a}$ are regular when we choose $\epsilon=1$. Since this vector corresponds to trajectories that travel at $45^{\circ}$ from right to left, all the horizon lines that are perpendicular to it necessitate to be dealt with the Kerr-Schild coordinates with the choice $\epsilon=1$. A similar reasoning with vector $S^{a}$ shows that all the other 
horizon crossings have to be dealt by using $\epsilon=-1$. We therefore have the following choices of $\epsilon$ for all the possible horizon crossings:

$$
\begin{gathered}
\epsilon_{1 \rightarrow 2}=\epsilon_{2 \rightarrow 5}=\epsilon_{6 \rightarrow 12}=\epsilon_{12 \rightarrow 11}=1, \\
\epsilon_{3 \rightarrow 2}=\epsilon_{2 \rightarrow 6}=\epsilon_{5 \rightarrow 12}=\epsilon_{12 \rightarrow 9}=-1 .
\end{gathered}
$$

And of course, the same applies for any transform whose both starting and ending regions are shifted by $8 k$, where $k$ is an integer, i.e. the crossing $4 \rightarrow 1$ is made with $\epsilon=-1$.

\subsection{The different types of geodesics}

If we want to visualize the Kerr metric, we need to solve the geodesic equation. Given the complexity of the full analytic extension of the metric, and given the fact that different coordinate systems may (and, actually, have to) be used when crossing several regions, it is hardly possible to propagate a geodesic by solving the geodesic equation by brute force if we do not know in advance which regions will be crossed by a geodesic whose position $x_{0}^{\mu}$ and four-velocity/wavevector $u_{0}^{\mu}, k_{0}^{\mu}$ are known at some event of the metric. Since there exists a closed form for $\dot{r}$ (see Eq. (17)), a geodesic can have at most two turning points, depending on the roots of $R(r)$ and on where $r_{0}$ is situated with respect to them. There are essentially five possible configurations, some of them possessing several sub-cases.

(1) Geodesics starting from $r=+\infty$ and with one turning point. Those are the only geodesics that exist when there is not black hole (or wormhole, in this context). In the presence of a wormhole, such geodesics can be divided into several sub-types, depending on where their turning point $r_{\mathrm{t}}$ lies.

(a) $\boldsymbol{r}_{\mathbf{t}}>\boldsymbol{r}_{+}$. The turning point is above the (outer) horizon, so that the geodesic never leaves its region of origin (region 1, say). These geodesics exist in the black hole case (and even in the absence of black hole). They may be called flyby geodesic: ${ }^{c}$

(b) $0<r_{\mathbf{t}}<r_{-}$. These geodesics cross the outer then inner horizon, bounce at some positive $r$ and then return back to another asymptotic region. We shall call them crossing geodesics. The geodesic trajectory within the outer horizon is not uniquely defined by the above constraint. Indeed, Eqns. 42 43. show that the coordinate change from Boyer-Lindquist $(t, \varphi)$ to Kerr-Schild $(T, \tilde{\varphi})$ can be made in order to deal with horizon crossing only by choosing the suitable value of $\epsilon= \pm 1$. These equations immediately show that when leaving their asymptotic region of origin (region 1, say) toward the interhorizon region (here, region 2), the fact that $\dot{r}$ is then negative implies that

\footnotetext{
${ }^{\mathrm{c}}$ It seems that there is no consensus about this naming. For example, Ref. 20 use the term of "flyby" for any geodesic with a turning point situated of some positive $r$ even though it occurs within the horizon. In our opinion, such convention is ambiguous since, although it is a flyby of the singularity itself, it is not necessarily a flyby of the wormhole.
} 
the only acceptable choice of $\epsilon$ is $\epsilon=\operatorname{sgn}\left(\left(r^{2}+a^{2}\right) E-a L_{z}\right)$, which, at horizon is rewritten

$$
\epsilon_{\text {outer,ingoing }}=\operatorname{sgn}\left(2 M r_{+} E-a L_{z}\right)
$$

The very same reasoning says that when leaving region 2 in order to cross the inner horizon, the correct choice of $\epsilon$ is then

$$
\epsilon_{\text {inner,ingoing }}=\operatorname{sgn}\left(2 M r_{-} E-a L_{z}\right) \text {. }
$$

The same reasoning shows that when crossing out the two horizon the choice of $\epsilon$ is the opposite:

$$
\begin{aligned}
& \epsilon_{\text {inner,outgoing }}=-\operatorname{sgn}\left(2 M r_{-} E-a L_{z}\right)=-\epsilon_{\text {inner,ingoing }}, \\
& \epsilon_{\text {outer,outgoing }}=-\operatorname{sgn}\left(2 M r_{+} E-a L_{z}\right)=-\epsilon_{\text {outer,ingoing }} .
\end{aligned}
$$

However, there is no reason that $\epsilon_{\text {outer,ingoing }}=\epsilon_{\text {inner,ingoing }}$ for geodesics originating from region 1 past null infinity, $E$ is positive, so that this equality is satisfied when $a L_{z} / E<2 M r_{-}$or $a L_{z}>2 M r_{+}$and is not satisfied when $2 M r_{-}<L_{z} / E<2 M r_{+}$. These two cases are met for geodesics crossing the horizon as can be seen from the reasonably well-known case of null equatorial geodesics which, in the extremal $a=M$, do cross the horizon when $-7 M<$ $L_{z} / E<2 M$ (see Ref. ${ }^{12}$ or next Section), a situation that encompass both cases mentioned above. Moreover, a geodesic leaving region 1 must end into another asymptotic region where $t$ is a future-oriented coordinate without experiencing more than one turning point, which leaves region 9 as its only possible destination. Consequently, these geodesics either travel through the $1,2,5,12,9$ or the $1,2,6,12,9$ sequences. In the first case, the same value of $\epsilon$ must be used for the first two (ingoing) horizon crossings and must be changed after these, whereas in the second case, the sign of $\epsilon$ must be changed at each horizon crossing.

(c) $\boldsymbol{r}_{\mathbf{t}}<\mathbf{0}$. Those geodesics that we may dub as adventurous have their turning point within a region of negative $r$. Because there is no ergoregion in any of the $r<0$ region, the region into which the geodesic can enter must have its $t$-coordinate that has the same orientation as its asymptotic region of origin, which, in the case the starting point is region 1 makes region 7 as its only negative $r$ region it can enter into. Therefore, the geodesic follows the sequence $1,2,5,7,5,12,9$. According to the previous discussion, there is no change of $\epsilon$ when traveling region 1 to region 2 and from region 2 form region 5 , however, as we shall explain later it is necessary to switch to Cartesian Kerr-Schild coordinate during the two crossings $5 \rightarrow 7$ and $7 \rightarrow 5$, the choice of $\epsilon$ being arbitrary at this stage (but a change of sign of $\epsilon$ will be necessary for the $5 \rightarrow 12$ crossing as compared to the $2 \rightarrow 5$ one, see previous sub-case above). Whether it is performed before, after or in between the two $5 \leftrightarrow 7$ crossings does not matter, however. 
(2) Geodesics starting from $r=-\infty$ and with one turning point. Those are the analog of the above, except that they start and end on the negative $r$ region (region -1 , say). Although there may be several sub-cases as in the above case depending on where the turning point lies, there is actually only one possible configuration, where the turning point is situated at some negative $r$. The reason for this is that should the geodesic be allowed to enter into a positive $r$ region and leave it afterward, this would mean that is lowest real root of $R(r)$ is positive. From Eq. (17), it is clear that the sum of the roots is zero, therefore there cannot be four positive roots, and in the case we are considering, there must be two positive roots and two complex conjugate roots with a negative real part. Let us call the positive roots $X-\delta, X+\delta$ and the two others $-X \pm i Y$, with $0 \leq \delta<X$. The coefficient of $R(r)$ that is proportional to $r$ is, in this case, $-2 X\left(Y^{2}+\delta^{2}\right)$. However, this coefficient is also equal to $2 M C$, where the Carter constant must be positive for $\dot{\theta}$ to be defined in Eq. 18), which implies that $X$ must be negative, which contradict the initial statement. Therefore, null geodesics starting and ending at $r=-\infty$ are of flyby type with a turning point at some negative $r$. They do not cross the ring singularity and remain in the same region they originate from.

(3) Ingoing transit geodesics. These geodesics start from $r=+\infty$ and end to $r=$ $-\infty$. In order to do so, their initial and final region must at the same time orientation for the $t$-coordinate and cannot have any turning point. Therefore, if they start from region 1 , they will experience the sequence $1,2,5,7$. Their mirror analogue stating from region 3 will go through the sequence $3,2,6,8$.

(4) Outgoing transit geodesics. Those are the affine parameter-reversed of the above. From the previous discussion, those that are seen by an observer in region 1 have gone through the sequence of regions $-1,-3,4,1$. For an observer in region 3, they must originate from region 0 .

(5) Bounded geodesics. These geodesics exist when the polynomial $R(r)$ admits four real roots, the geodesics being bounded between the two intermediate roots. We shall devote a thorough analysis of these geodesics in the next Section, but we may already summarize the results: those geodesics can be seen in a rather short interval of $r$, that cannot exceed $[0,4 M]$. For the same reason that is explained in the case of crossing geodesics, a bounded geodesic that travels at some point within region 1 can further go, after outer then inner horizon crossing, either in region 5 or 6 . Therefore, such geodesics can cross four types of infinite sequences: $1,2,5,12,9, \ldots[\bmod 8], 1,2,6,12,9, \ldots[\bmod 8]$, $3,2,5,12,11, \ldots[\bmod 8]$ and $3,2,6,12,11, \ldots[\bmod 8]$.

The above discussion allows to determine which regions are seen by an observer as a function of its position. This is summarized in Table 1. One (we think unexpected) consequence of the global structure of geodesics is that the richest patch of the Kerr wormhole is the outgoing inter-horizon region (i.e., region 12, ( $\bmod 8$, of course) which allows to see both two negative $r$ regions as well as two positive $r$ 
asymptotic regions, a situation which is not symmetric with respect to ingoing interhorizon region, where no geodesic starting from any past null infinity of negative $r$ region penetrate into.

Table 1. Summary of the asymptotic regions that can be seen as a function of the region of the Carter-Penrose diagram where the observe lies. For each of the region that lie one one side of the diagram, we have also included the view from its mirror counterpart of the diagram. All the label of given line can be simultaneously shifted by $8 k$. BG means that some bounded geodesics can be seen by the observer (see next Section).

1: Bounded geodesics are seen for sufficiently small $r$;

2: Bounded geodesics are always seen;

3: Up to two bounded geodesic patches can be seen, depending on the observer's coordinates $r$ and $\theta$.

\begin{tabular}{|c|c|c|}
\hline Region where the observer lies & Type & Region that are seen \\
\hline 1 (resp. 3) & Asymptotic, positive $r$ & $1,-7,-1($ resp. $3,-5,0)+$ BG $^{1}$ \\
2 & Ingoing inter-horizon & $1,3+\mathrm{BG}^{2}$ \\
5 (resp. 6) & Within inner horizon, positive $r$ & $1,3,7$ (resp. 3, 1, 8) + BG $(2 \times)^{3}$ \\
7 (resp. 8) & Negative $r$ & 7,1 (resp. 8, 3) \\
12 & Outgoing inter-horizon & $1,3,7,8+\mathrm{BG}^{2}$ \\
\hline
\end{tabular}

\section{Bounded geodesics}

In our opinion, the most overlooked aspect of geodesics in the Kerr metric deals with bounded null geodesics. Those are essential to be taken into account when visualizing the metric in region where they exist. The problem they pose becomes necessary to address for any observer sufficiently close to the black hole outer horizon as we shall see.

The function $R(r)$ is a fourth degree polynomial and therefore can admit up to four roots. Consequently, it is possible that a geodesic, whether timelike or null, is bounded. For visualization purpose, we shall only consider null geodesics which share this property. For null geodesics, $\kappa=0$, and the three other constants of motions are defined up to some arbitrary overall constant since the geodesic affine parameter is as well. Unless we have to deal with a very peculiar geodesic with $E=0$, it is convenient to rescale the constants of motion so as to reduce them to two: $L_{z} / E$ and $C / E^{2}$. Moreover, the latter is more conveniently replaced by $\left(C-\left(a E-L_{z}\right)^{2}\right) / E^{2}$. Keeping the notations of Ref. ${ }^{12}$, we therefore define

$$
\begin{aligned}
\xi & :=\frac{L_{z}}{E}, \\
\eta & :=\frac{C}{E^{2}}-(a-\xi)^{2} .
\end{aligned}
$$

With these notations, $R(r)$ can be rewritten in the case of null geodesics as

$$
\left.\frac{R(r)}{E^{2}}=r^{4}-\left[\eta+\xi^{2}-a^{2}\right] r^{2}+2 M\left[\eta+(a-\xi)^{2}\right)\right] r-a^{2} \eta
$$

In the $(\xi, \eta)$ parameter space, the edge of the bounded geodesic region arises when $R(r)$ admits a double root that we shall label $e$, i.e., when one has simultaneously 
$R(e)=0$ and $R^{\prime}(e)=0$. Writing these two equations and using one of them to express $\eta$ as a function of $\xi$ and $e$ and further solving the second order equation for $\xi$ yields a parametric equation for both $\xi$ and $\eta$ as a function of the double root $e$

$$
\begin{aligned}
& \xi=\frac{1}{a(e-M)}\left[M\left(e^{2}-a^{2}\right)-e \Delta(e)\right], \\
& \eta=\frac{e^{3}}{a^{2}(e-M)^{2}}\left[4 M a^{2}-e(e-3 M)^{2}\right] .
\end{aligned}
$$

By definition, these two equations separate the loci of some pairs of geodesic types among the five of 4.6 of the previous Section. This is of course the case for any type of metric where a closed form for $\dot{r}$ exists. For example, in the case where $a$ tends to $0, \eta$ and $\xi$ remain defined only when their numerator tends to 0 as well, which occurs only for $e=3 M$. We recover the critical case of null geodesics in the Schwarzschild metric which separates between the three types of geodesics that exist in this metric (flyby geodesics, which have a turning point at some $r>3 M$, transit geodesics which go from infinity to $r=0$ or vice-versa and geodesics starting and ending at the singularity). What we need to do now is to narrow this constraint to consider when they refer to bounded geodesics only.

Although not very illuminating, Eq. 62 for $\xi(e)$ can be rewritten in several ways which can occasionally shed more light on these expressions. For example, we have

$$
\begin{aligned}
\xi & =a-\frac{e}{a(e-M)}\left[e^{2}-3 M e+2 a^{2}\right], \\
\xi-a & =-\frac{e}{a(e-M)}\left[e(e-3 M)+2 a^{2}\right], \\
\xi+a & =-\frac{1}{a(e-M)}\left[e^{2}(e-3 M)+2 M a^{2}\right] .
\end{aligned}
$$

The last two equations can be combined with Eq. (63) to obtain

$$
\begin{aligned}
\eta+\xi^{2}-a^{2} & =2 e^{2}+\frac{4 M e \Delta(e)}{(e-M)^{2}}, \\
& =\frac{2 e}{(e-M)^{2}}\left[e^{3}-3 M^{2} e+2 M a^{2}\right],
\end{aligned}
$$

together with

$$
\begin{aligned}
\eta+(\xi-a)^{2} & =\frac{4 e^{2} \Delta(e)}{(e-M)^{2}}, \\
\eta+(\xi+a)^{2} & =\frac{4 M}{(e-M)^{2}}\left[2 e^{3}-3 M e^{2}+M a^{2}\right] .
\end{aligned}
$$

Finally, Eq. 63 alone yields

$$
a^{2} \eta+e^{4}=\frac{4 M e^{3}}{(e-M)^{2}} \Delta(e) .
$$


The sum of the roots of $R$ is 0 and their product is $-a^{2} \eta$, therefore, since when $R$ admits $e$ as a double root, it can necessarily be rewritten according to

$$
R(r)=(r-e)^{2}\left(r^{2}+2 r e-a^{2} \eta / e^{2}\right) .
$$

In order to have bounded geodesics, there must be two other roots in $R$, otherwise the double root only separates between geodesics that start from $\pm \infty$ and have a finite turning point before going back to $\pm \infty$, and geodesics that cross the whole interval of $r$, i.e. from $-\infty$ to $+\infty$ or vice versa. The discriminant of the second order polynomial in the right-hand side of Eq. $(72)$ is $4\left(e^{4}+a^{2} \eta\right) / e^{2}$. Using Eq. (71), it is therefore clear that the two mandatory other roots are defined only when $e \Delta(e)>0$, something that happens either when $0 \leq e \leq r_{-}$or $r_{+} \leq e$. When one of these to conditions is satisfied, we shall label $s$ the largest of the two roots, the other being $-s-2 e$. These roots are given by

$$
s,-2 e-s=-e \pm \frac{2}{|e-M|} \sqrt{M e \Delta(e)}
$$

where the plus sign of the right-hand side corresponds to $s$. For $e>M$, one immediately sees that $s<e$, which comes from the evident inequality $(e-M)^{3}+M\left(M^{2}-\right.$ $\left.a^{2}\right)>0$. When $e<M$, which in this context happens when $0 \leq e \leq r_{-}, s$ is smaller than $e$ in a fairly limited interval whose lower bound is given by the equality $s=e$, which occurs when $(M-e)^{3}=M\left(M^{2}-a^{2}\right)$ whose unique real solution is

$$
e_{\min }=M-\left[M\left(M^{2}-a^{2}\right)\right]^{\frac{1}{3}} .
$$

Equivalently, this $e_{\min }$ can be found by noting that the double root remains a local maximum as long as $R^{\prime \prime}(e)<0$. When $e<s$, e becomes a local minimum, the transition between the two occurring when $R^{\prime \prime}\left(e_{\min }\right)=0$, whose solution, according to the definition of Eq. 61) occurs when $12 e_{\min }^{2}=2\left(\eta\left(e_{\min }\right)+\xi^{2}\left(e_{\min }\right)-a^{2}\right)$; whose solution is given by the same equation as that of Eq. 74.

What we know need is to determine whether Eqns. 62 63) can actually correspond to geodesics, that is to check which values of $\theta$ (if any) are compatible with these requirements. By virtue of Eq. (18), we have, for null geodesics,

$$
-\sin ^{2} \theta \frac{\Sigma^{2} \dot{\theta}^{2}}{E^{2}}=P\left(\mu^{2}\right)=a^{2} \mu^{4}+\left(\eta+\xi^{2}-a^{2}\right) \mu^{2}-\eta<0,
$$

where we have set, following the usual convention, $\mu=\cos \theta$. This inequality can be satisfied only when the corresponding second order polynomial in $\mu^{2}$ admits real roots, i.e., when the corresponding discriminant is positive.

This discriminant, $\Delta^{\prime}$, can be written

$$
\Delta^{\prime}=\left(\eta+\xi^{2}-a^{2}\right)^{2}+4 a^{2} \eta=\left(\eta+(\xi-a)^{2}\right)\left(\eta+(\xi+a)^{2}\right) .
$$

Let us consider the function $g_{3}(e)=2 e^{3}-3 M e^{2}+M a^{2}$, whose sign is the same as $\eta+(\xi+a)^{2}$ for each value of $e$, see Eq. 70 . Whichever value of $a$, the function $g_{3}$ possesses two extrema at $e=0, M$ and is therefore increasing for $e<0$ and $e>M$, and decreasing for $0<e<M$. Moreover, for any $a$ such that $0<a^{2}<M^{2}$, we 
have $g_{3}(0)=M a^{2}>0$ and $g_{3}(M)=-M\left(M^{2}-a^{2}\right)<0$. Consequently, $g_{3}$ admits three roots, $r_{1}, r_{2}$ and $r_{3}$, such that $r_{1}<0<r_{2}<M<r_{3}$. In the limit case $a=0$, we have $r_{1}=r_{2}=0$ and when $a^{2}=M^{2}$, we have $r_{2}=r_{3}=M$. Moreover, we also have $g_{3}\left(r_{ \pm}\right)=2 r_{ \pm}\left(M^{2}-a^{2}\right)>0$, so that we have in fact

$$
r_{1}<0<r_{-}<r_{2}<M<r_{3}<r_{+} .
$$

Consequently, the discriminant $\Delta^{\prime}$ is positive within the three intervals $] r_{1}, r_{-}[$, ]$r_{2}, r_{3}[$ and $] r_{+},+\infty\left[\right.$, and it is zero at these five $r_{i}$ 's.

The fact that the discriminant is positive is not sufficient. In addition, the interval between the two roots of the polynomial $P\left(\mu^{2}\right)$ of Eq. 75$)$ must have some intersection with interval $[0,1]$ where $\mu^{2}$ is defined.

From Eq. 75), it is clear that there are viable solutions as soon as the product of the roots is negative, that is, when $\eta$ is positive. Conversely, if the product of the roots is positive (i.e., $\eta<0$ ) whereas their sum is negative, then there are no solutions to Eq. 75).

For positive $e$ 's, the sign of $\eta$ is given by that of the function $f_{3}(e)=4 M a^{2}-e(e-$ $3 M)^{2}$ (see Eq. (63)). This function appears in a well-known context when one studies the Kerr metric: its roots correspond to the radii of equatorial, null geodesics. This functions admits two extrema at $e=M, 3 M$. Moreover, since $f_{3}(0)=4 M a^{2}>0$, $f_{3}(M)=4 M\left(a^{2}-M^{2}\right)<0$ and $f_{3}(3 M)=4 M a^{2}<0, f_{3}$ admits three roots, we shall label $r_{\mathrm{i}}, r_{\mathrm{p}}$ and $r_{\mathrm{r}}$ which lies within the intervals $] 0, M[] M,, 3 M[$ and ] $3 M, \infty\left[\right.$, respectively. Using the fact that $r_{ \pm}^{2}=2 M r_{ \pm}-a^{2}$, it is easy to show that $f_{3}\left(r_{ \pm}\right)=-r_{ \pm}\left(M^{2}-a^{2}\right)<0$, which means that we have in fact $0<r_{\mathrm{i}}<r_{-}$and $r_{+}<r_{\mathrm{p}}<3 M$. Furthermore, if we use the definition of $e_{\mathrm{min}}$, we can easily show (by canceling terms proportional to $\left.a^{2}\right)$ that $f_{3}\left(e_{\min }\right)=3 e_{\min }\left(e_{\min }-M\right)^{2}>0$, which ensures that $e_{\min }<e_{\mathrm{i}}$.

All this enables to extend Eq. (77) into

$$
r_{1}<0<r_{\mathrm{i}}<r_{-}<r_{2}<M<r_{3}<r_{+}<r_{\mathrm{p}}<3 M<r_{\mathrm{r}} .
$$

For the sake of completeness, we recall that the equatorial null geodesics occur at

$$
r_{\text {eq,null }}=2 M[1+\cos (2 \arccos (a / M) / 3+2 k \pi / 3)],
$$

with $k=0,1,2$ for $r_{\mathrm{r}}, r_{\mathrm{i}}$ and $r_{\mathrm{p}}$, respectively. The corresponding value of $\xi$ is given by

$$
\xi_{\text {eq,null }}=-a-6 M \cos \left[\frac{1}{3} \arccos \left(\frac{a}{M}\right)-\frac{2 k \pi}{3}\right],
$$

where we have defined $k$ in a consistent way between Eqns. 79 and 80 .

Let us now consider the quantity $\eta+\xi^{2}-a^{2}$, which is the opposite of the sum of the roots of Eq. 775). It is of same sign as $e\left(e^{3}-3 M e+2 M a^{2}\right):=e h_{3}(e)$. The third order polynomial $h_{3}(e)$ admits two extrema at $e= \pm M$, and $h_{3}(-M)=$ $2 M\left(M^{2}+a^{2}\right)>0, h_{3}(M)=-2 M\left(M^{2}-a^{2}\right)<0$. Also, $h_{3}(0)=2 M a^{2}>0$. Consequently, $h_{3}$ possesses three roots, one lower than $-M$ and two positive ones. 
Moreover, $h_{3}\left(r_{ \pm}\right)=r_{ \pm}\left(M^{2}-a^{2}\right)>0$, so that the two positive roots of $h_{3}$ are between $r_{-}$and $r_{+}$. If we consider $h_{3}\left(r_{1}\right)$, where $r_{1}$ is the negative root of $g_{3}$, we have immediately $h_{3}\left(r_{1}\right)=-r_{1}^{3}+M a^{2}>0$, so that $r_{1}$ is larger that the negative root of $h_{3}$, and $h_{3}(r)$ is positive everywhere in the interval $\left[r_{1}, 0\right]$. Conversely, $\eta+\xi^{2}-a^{2}$ is negative. We can now summarize the domain of existence of a double root $e$ of $R$ which delineates the edge of a bounded geodesic. It must fill the following requirements:

(1) Some roots must exist to polynomial $P\left(\mu^{2}\right)$ in Eq. 75$)$, i.e. discriminant $\Delta^{\prime}$ must be positive;

(2) The roots of $P\left(\mu^{2}\right)$ must be such that they span an interval that has a non zero intersection with physically allowed values for $\mu^{2}$, i.e., $[0,1]$;

(3) The turning point $e$ must be outside the outer horizon or inside the inner horizon;

(4) There must be two other real roots, i.e., according to Eq. (73), one must have $e \Delta(e)>0$

(5) The double root must be a local maximum.

All these requirements are summarized into Table 2 from which we see that acceptable values for $e$ are those which lies within $\left[0, r_{\mathrm{i}}\right]$ and $\left[r_{\mathrm{p}}, r_{\mathrm{r}}\right]$. Within these two intervals, both $\eta$ and $\eta+\xi^{2}-a^{2}$ are positive, which means that Eq. (75) admits one positive and one negative solution. Consequently, bounded null geodesics for which $e$ is defined all oscillate around the equatorial plane and reach a maximum value of $\mu^{2}$ defined by

$$
\mu_{\max }^{2}=\frac{e}{a^{2}(e-M)^{2}}\left[-\left(e^{3}-3 M^{2} e+2 M a^{2}\right)+\sqrt{4 M \Delta(e)\left(2 e^{3}-3 M e^{2}+M a^{2}\right)}\right] .
$$

One can check that this expression reaches 1 only when $e$ is a root of Eq. (64), i.e. when $\xi=0$ and for $e=r_{\text {pol }}$. Another useful value is when $e=e_{\min }$, for which Eq. (81) simplifies considerably into

$$
\mu_{\max }^{2}\left(e_{\min }\right)=(2 \sqrt{3}-3) \frac{e_{\min }^{2}}{a^{2}} .
$$

Although we already know that it is the case, we can check that this quantity is smaller than 1 since the ratio $e_{\min } / a$ is already.

The value of the solutions to Eq. (75) are shown in Figure 8 .

An equatorial observer will intersect bounded null geodesics at $r=r_{\mathrm{r}}$ and below, but this will be not the case for a non equatorial observer. Such observer lying somewhere in the interval $r \in\left[r_{\mathrm{p}}, r_{\mathrm{r}}\right]$ will actually intersect bounded null geodesics only if such geodesics can reach the observer given its colatitude $\theta$, that is if the observer's colatitude lies with the range allowed by Eq. (75). For example, an observer which lies along the rotation axis of the black hole will intersect bounded null geodesics only if those are polar, that is if $\xi(e)=0$. Given that, according to Eq. 62), $\xi(e) \propto j_{3}(e)=M\left(e^{2}-a^{2}\right)-e \Delta(e)=-e^{3}+3 M e^{2}-a^{2} e-M a^{2}$, we need 
Table 2. Intervals where double roots to $R$ exist and delineate edge of bounded geodesics, according to Eq. 75. Intervals where it is not necessary to define $\eta+\xi^{2}-a^{2}$ are labeled with a question mark.

1: No root since $\Delta^{\prime}<0$

2: No acceptable root as their sum $\left(-\eta-\xi^{2}+a^{2}\right)$ is negative and their product $(-\eta)$ is positive

3: No root allowed since no geodesic turning point can exist between $r_{-}$and $r_{+}$and, equivalently, $C=\Sigma^{2} \dot{\theta}^{2}+\left(a E \sin \theta-L_{z} / \sin \theta\right)^{2}<0$

4: No other roots apart from the double root

5: Double root is not a local maximum

\begin{tabular}{|c|c|c|c|c|c|c|c|c|c|c|c|c|c|c|c|c|c|c|c|c|c|c|c|}
\hline & & $r_{1}$ & & 0 & & $e_{\min }$ & $r_{\mathrm{i}}$ & & $r_{-}$ & & $r_{2}$ & & $M$ & & $r_{3}$ & & $r_{+}$ & & $r_{\mathrm{p}}$ & & & $r_{\mathrm{r}}$ & \\
\hline$\Delta(r)$ & + & & + & & + & + & & + & 0 & - & & - & & - & & - & 0 & + & & + & + & & + \\
\hline$\eta+(\xi-a)^{2}$ & + & & + & 0 & + & + & & + & 0 & - & & - & II & - & & - & 0 & + & & + & + & & + \\
\hline$\eta+(\xi+a)^{2}$ & - & 0 & + & & + & + & & + & & + & 0 & - & & - & 0 & + & & + & & + & + & & + \\
\hline$\Delta^{\prime}$ & - & 0 & + & 0 & + & + & & + & 0 & - & 0 & + & 11 & + & 0 & - & 0 & + & & + & + & & + \\
\hline$\eta$ & - & & - & 0 & + & + & 0 & - & & - & & - & $\|$ & - & & - & & - & 0 & + & + & 0 & - \\
\hline$\eta+\xi^{2}-a^{2}$ & $?$ & & - & 0 & + & + & & + & & $?$ & & $?$ & $\|$ & ? & & $?$ & & + & & + & + & & + \\
\hline Some $\mu^{2}$ defined & No & & No & & No & Yes & & No & & No & & No & & No & & No & & No & & Yes & Yes & & No \\
\hline Notes & $1,4,5$ & & 4,5 & & 5 & & & 2 & & $1,3,4$ & & 3,4 & & 3,4 & & $1,3,4$ & & 2 & & & & & 2 \\
\hline
\end{tabular}

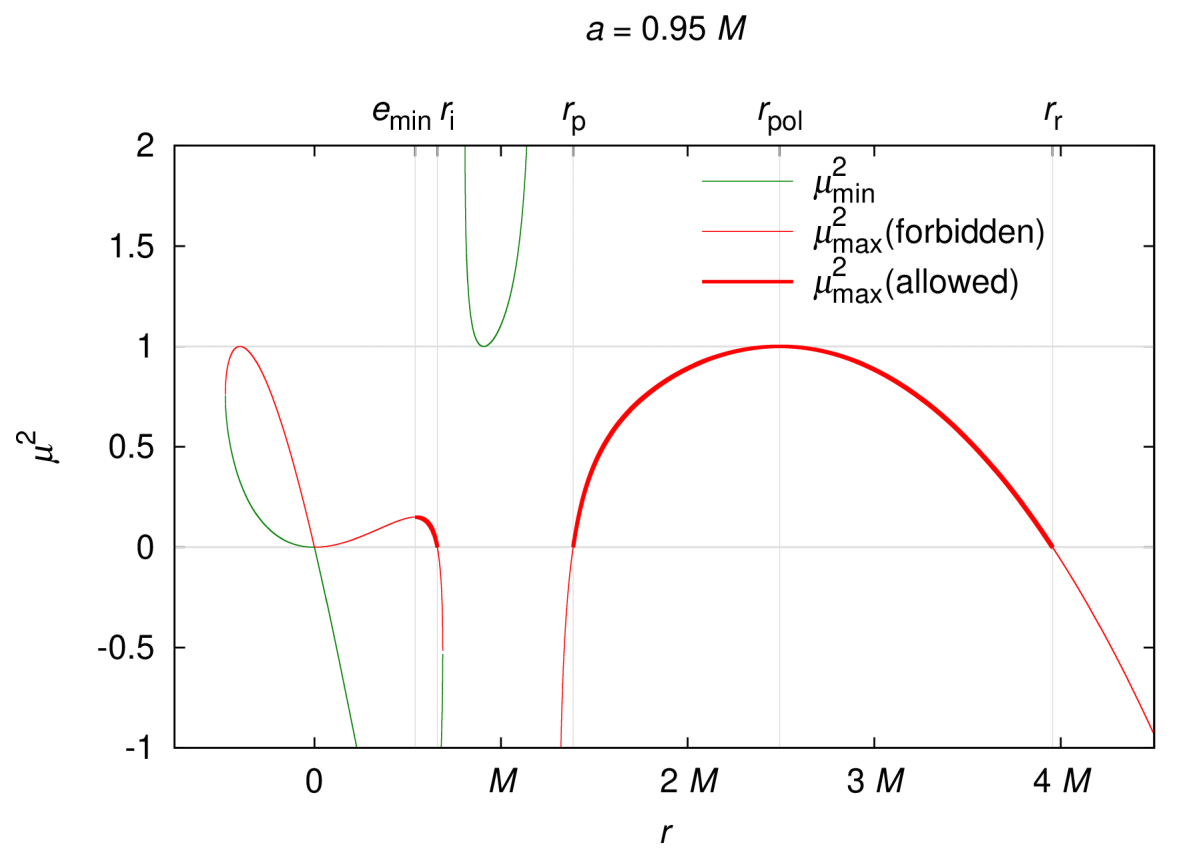

Fig. 8. Roots of polynomial $P\left(\mu^{2}\right)$ defined in Eq. 75 as a function of the double root of $R(r)$. See text for the physical meaning of this function. The overall shape of the function does not depend on $a$ (as long as $a^{2}<M^{2}$, of course), however, the small part that lies between 0 and $r_{\mathrm{i}}$ is extremely small and has a low vertical extension and, hence, difficult to see unless one considers a value of $|a|$ close to $M$, which is the reason why we chose $a=0.95 M$ here.

to find where the roots of $j_{3}$ lie. From the first expression of $j_{3}$, one obtains that $j_{3}\left(r_{ \pm}\right)=M\left(r_{ \pm}^{2}-a^{2}\right)=2 M\left(M r_{ \pm}-a^{2}\right)=2 M r_{ \pm}\left(r_{ \pm}-M\right)$, so that $j_{3}\left(r_{-}\right)<0$ and $j_{3}\left(r_{+}\right)>0$. Since, in addition, $j_{3}(e)$ is decreasing for sufficiently large $|e|$ and $j_{3}(0)<0, j_{3}$ evidently admits three roots, a negative one, one between $r_{-}$and 
$r_{+}$and one, we shall label $r_{\text {pol }}$, above $r_{+}$. Direct investigation of Eq. 80 show that $\xi\left(r_{\mathrm{p}}\right)+a \in[3 M, 3 \sqrt{3} M]$, whereas $\xi\left(r_{\mathrm{r}}\right)+a \in[-6 M,-3 \sqrt{3} M]$, which ensures that $r_{\mathrm{pol}}$ lies between $r_{\mathrm{p}}$ and $r_{\mathrm{r}}$, an unsurprising result since the latter corresponds to an equatorial, retrograde orbit (hence with a negative $\xi$ ) and the former to an equatorial, prograde orbit (hence with a positive $\xi$ ).

Consequently, an observer falling toward the black hole will begin to intersect bounded null geodesics at some $r$ between $r_{\mathrm{r}}$ and $r_{\mathrm{pol}}$ depending on how its colatitude evolves with $r$. If the observer always lies along the equatorial plane, bounded null geodesics will be intercepted as early as $r=r_{\mathrm{r}}$, and as late as $r=r_{\text {pol }}$ for a polar observer (i.e., always along the black hole rotation axis). The value of $r_{\text {pol }}$ is given by

$$
r_{\text {pol }}=M+2 \sqrt{M^{2}-a^{2} / 3} \cos \left(\frac{1}{3} \arccos \left[M\left(M^{2}-a^{2}\right) /\left(M^{2}-a^{2} / 3\right)^{\frac{3}{2}}\right]\right),
$$

a quantity which is $3 M$ for $a=0$ (as expected since it corresponds to the unstable circular photon orbits of the Schwarzschild case) and which is otherwise always smaller than $3 M$.

\section{Dark crescent, dark outgrowth, dark shell, dark bubble}

We now come to the visual translation of the above discussion regarding the aspect of bounded geodesics.

When considering the black hole case, these bounded null geodesics are, from a visual point of view, indistinguishable from the black hole silhouette itself. Actually, they do delineate the black hole silhouette because all these geodesics have crossed out the horizon before reaching the observer, so that they can be considered as originating from a perfectly black (actually infinitely redshifted) surface. However, if we consider the full analytical extension of the metric, there is a fundamental distinction between bounded and unbounded geodesics.

Let us first consider an observer situated on the equator of the coordinate system at coordinate distance $r$. The observer's motion does not matter here, since switching from a given observer to another observer endowed with another fourvelocity will translate into an aberration transformation due to the corresponding Lorentz boost, and because such distortion of the celestial sphere does not affect the topology of the patches that are on that celestial sphere. Let us suppose that the observer starts from a large value of $r$ of its region of origin, region 1 (say). As long as $r>r_{\mathrm{r}}$, there are two type of null geodesics the observer can cross. Either the null geodesic originates from past null infinity of the observer's region, in which case they will show some part of region 1, or the geodesic originates from some region that lies in the past lightcone of the observer, which, according to the discussion of 4.1 is either -7 or -1 . Forgetting about the latter, the edge between direction where regions 1 and -7 are given by unstable null geodesics, each of which lie at some fixed $e$ whose value is given by the double roots of $R(r)$ which are a local maximum. 
Let us consider geodesics showing region 1 close to this boundary. These geodesics have constants of motion close to $\xi(e), \eta(e)$, the departure from these values being such that the local extremum of $R$ is no longer a double root but a (very) slightly positive extremum. Those geodesics are seen after they have reached their lowest approach $r$ coordinate (that is, $e$ ) and they are now receding away from the wormhole. The same applies for geodesics originating from region -7 except that now the corresponding constants of motion, although also close to $\xi(e), \eta(e)$ are this time such that the local extremum of $R$ is no longer a double root but a (very) slightly negative extremum, which allowed these geodesics, when ingoing from region -7 to spend a long time close to $r=e$ (in region -7 ), then crossed the two outer, then inner horizons, bounced at some $r$ close to $s(e)$ either in region -3 or -2 , exited the inner then outer horizon through region 4 and spent a large amount of time close to $r=e$ in region 1 when they were outgoing. Those are therefore seen as well when they are outgoing.

When the observer reaches some $r$ only a bit smaller than $r_{\mathrm{r}}$, the situation changes. Focusing on equatorial geodesics, the equatorial null geodesic from region 1 past null infinity that has $e=r_{\mathrm{m}}$ as a double root is no longer seen by the observer, however the one with slightly different value of $\xi \eta$, for which $r=e$ is a negative local maximum of $R$, is seen whereas it is ingoing. Conversely, its analog from region -7 is still seen whereas it is, of course, outgoing. This means that these two geodesics are no longer seen along the same direction, because although they have identical (or almost identical) constants of motion, the former is seen as it is still ingoing, whereas the latter is seen when it is outgoing (i.e., if we consider BoyerLindquist coordinates, each of their $k^{\mu}$ components are equal to that of the other geodesic up to an overall normalization factor, except for the $r$ component, which changes sign). In the segment (along sky directions) that joins these two geodesics, we now see bounded geodesics. Consequently, the interior of the wormhole silhouette is going to be split into two parts: one which, as before, shows region -7 , and the other that corresponds to bounded geodesics which we assume to the devoid of any photon, and hence perfectly black d The angular size of this patch is exactly 0 as long at the observer lies at $r \geq r_{\mathrm{r}}$ and starts growing from this point. In the usual representation where the black hole is spinning counterclockwise if seen from above the equator, the dark patch appears on the right within the wormhole silhouette since the rightmost point of this silhouette is delineated by clockwise equatorial geodesics. Again when looking at the black hole or wormhole silhouette at some distance, the furthest to the left to point of this silhouette we are looking at, the lowest its closest approach point $e$ is in term of the $r$-coordinate. The upper and lower edge of the silhouette correspond to the polar null unstable geodesics which lies at $r=r_{\text {pol }}$ (see Eq. (83p), the leftmost part to equatorial prograde geodesics

\footnotetext{
${ }^{\mathrm{d}}$ One may dispute this choice. Photons travelling along bounded null geodesics may originate from any asymptotic region of the observer's past lightcone. One may therefore consider that looking towards these directions should instead show unpredictable patterns instead of a black patch.
} 
with turning point at $r_{\mathrm{p}}$.

As the observer decreases its $r$ coordinates, it is going to intercept bounded null geodesics which have excursions outside the equatorial plane according to the relation $\mu_{\max }(e)$, see Figure 8. Visually this will translate into the fact that the patch of bounded null geodesics will increase in size upward and downward along the inner part of the wormhole silhouette, while increasing in horizontal thickness. Indeed, if we consider for example the geodesics coming from regions 1 and -7 both endowed with constant of motion $\xi\left(r_{\mathrm{r}}\right), \eta\left(r_{\mathrm{r}}\right)$ they will be seen further and further from their $r$-coordinate "loitering point", so that that their opposite $k^{r}$ component will be increasingly different (opposite to another but with a larger absolute value). Conversely, the interior of the wormhole silhouette occupied by geodesics originating from region -7 will decrease in size accordingly. Numerical investigation show that the wormhole silhouette remains reasonably close to circular even when one reaches $r_{\mathrm{r}}$, and so does the now different patch that shows region -7 . Consequently, the patch of the celestial sphere spanned by bounded geodesics takes the shape of some crescent (of first crescent type if we refer to Moon phases seen from the northern hemisphere).

The topology of the scenery will change when the observer reaches $r=r_{\mathrm{p}}$. There and from now on, all the geodesics starting from region 1 past null infinity that delineate the wormhole silhouette are seen as they are ingoing, whereas all the geodesics that come from region -7 are seen outgoing. Therefore, none of the geodesics of the first set are seen, in term of angular distance, close to geodesics of the second set. In between those two sets, null geodesics that intersect observer's trajectory are bounded, therefore, they form a thick shell inside which region -7 is seen, a situation which is qualitatively similar of the Reissner-Nordström metric 21, except that here the patch showing region -7 is off-centere

The above situation will be also qualitatively the same for a non equatorial observer, the only difference being the value of the $r$-coordinate at which the different steps will begin. Assuming for simplicity that this new observer has a trajectory of decreasing $r$ but constant $\theta$, then the dark crescent will start of appear at the $r_{\text {cresc }}$ such that $\mu_{\max }\left(r_{\text {cresc }}\right)=\cos ^{2} \theta$, where we choose the largest value of $r$ satisfying this constraint (see Fig. 8). The dark crescent will transform into a dark shell at the second largest root, $r_{\text {shell }}$ of the equation $\mu_{\max }\left(r_{\text {shell }}\right)=\cos ^{2} \theta$. One special case arises in this context: a polar observer will see an immediate transition between region -7 and region 1 patches stuck together to them being separated by a dark shell at $r=r_{\text {pol }}$, just as this was the case for the Reissner-Nordström metric.

When going toward lower values of $r$, three other events plus two optional ones are worth mentioning. Among the first three, one occurs at outer horizon crossing, the two others after inner horizon crossing. The optional events occur also after inner horizon crossing (in case they actually do occur), prior to the last two certain ones, which we shall focus on first.

$\overline{\mathrm{e}}$ Although it can be put in-center by performing a Lorentz transformation. 
Firstly, when approaching the outer horizon, the patch showing region -7 is going to shrink to 0 . This can be understood as an extreme example of aberration, and/or as a consequence of the fact that entering into the outer horizon toward region 2 is equivalent to leaving region 1 which geodesics from region -7 could reach, whereas it is not the case for region 2. As soon as the observer enters region 2, region 3 enters into its past lightcone and hence become visible. Where region 3 appears is very easy to compute: if we consider a null geodesic coming from region -7 with given constants of motion $E, L_{z}$, and $C$, the direction along which it is seen by an observer of region 1 (where the geodesic is outgoing) is uniquely determined by the reduced constants of motion $\xi$ and $\eta$. Let us now consider a null geodesic coming from region 3. Its constant of motion $E^{\prime}$ is now negative, but obviously, the null geodesic with constants of motion $E^{\prime}=-E, L_{z}^{\prime}=-L_{z}$ and $C^{\prime}=C$ possesses the same reduced constants of motion, $\xi, \eta$ as the above mentioned geodesic coming from region -7 . The former, seen just before outer horizon crossing is therefore seen along the same direction as the latter, seen immediately after horizon crossing. Region 3 is therefore going to occupy a patch whose size is initially 0 and that shall grow afterward within the patch of bounded geodesics.

Secondly, there is a moment where the observer will reach $r_{\text {bubble }} \equiv s\left(r_{\text {shell }}\right)$. Since $r_{\text {shell }}$ was the starting point of the shell-type configuration, with patch of region -7 and then region 3 fully surrounded by the shell of bounded null geodesics, $s\left(r_{\text {shell }}\right)$ corresponds to the disappearance of the configuration, that is the (actually temporary) disappearance of region 3 . Such a configuration is not unexpected: except when the observer remains within the equatorial plane, decreasing $r$ while keeping its $\theta$ fixed makes the observer exit the inner ergoregion, within which region 3 could be seen from region 5 . Within region 5 but outside the inner ergoregion, there is no possibly to see region 3 . The patch of bounded null geodesics which was at this stage like a shell therefore transforms into a bubble, whose name is inspired by the fact that we noticed from the numerical simulations that this region was reasonably close to a disk (although slightly oblate for the configurations we studied).

Thirdly, we going even closer to $r=0$, the observer will reach $r_{\text {dis }} \equiv s\left(r_{\text {shell }}\right)$, which means that it will exit the region that can be reached by bounded null geodesics. In this case the patch on the celestial sphere which showed them will simply disappear.

As an option, if the observer is sufficiently close to the equatorial plane, more precisely if its (assumed constant) $\theta$ is such that $\cos ^{2} \theta<\mu_{\max }^{2}\left(e_{\min }\right)$ (see Eq. 82 ), then the observer will intersect a second set of null bounded geodesics, as early as $r_{\mathrm{i}}$ if it lies on the equatorial plane, and as late as $r=e_{\min }$ if it has the highest allowed latitude in order to do so, given by Eq. 82 .

The overall possibilities regarding the shape and topology of the bounded geodesic patches are summarized in Fig. 9. By looking at this Figure, it appears that for some $\mu^{2}=\cos ^{2} \theta$ of the observer, the topology of the bounded geodesics immediately changes from the dark shell to the dark bubble configuration at inner horizon crossing. Given the previous discussion, this amounts to say that among 


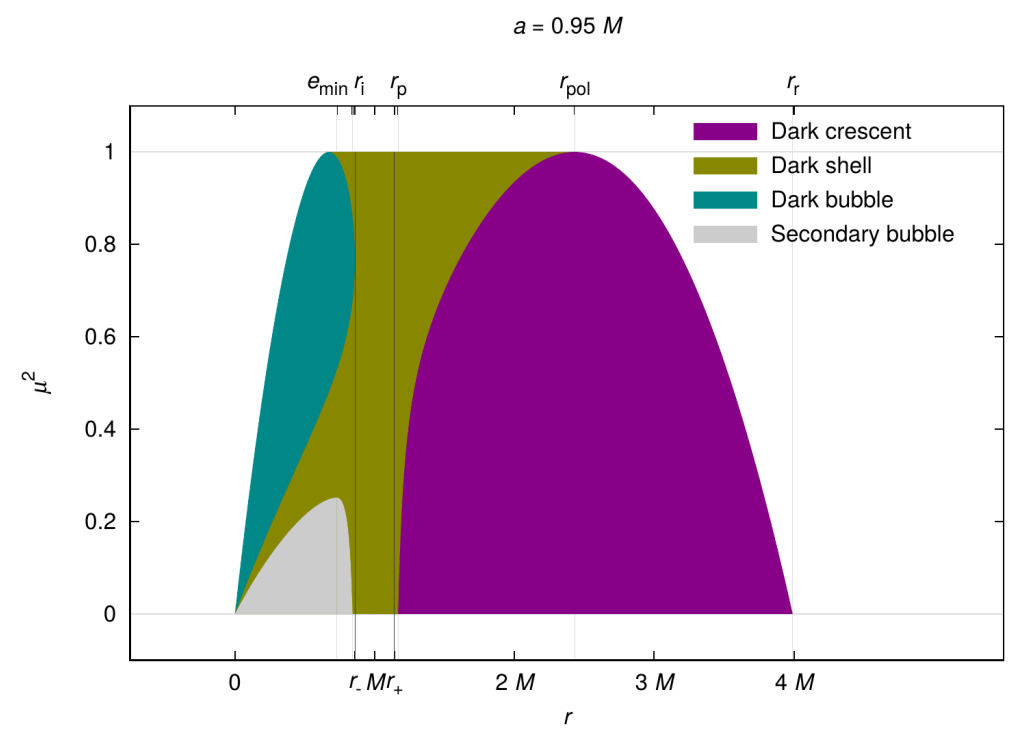

Fig. 9. Regions in the $(r, \theta)$ plane of the observer's position which show different types/topologies of the patch(es) occupied by bounded null geodesics. For any $r$ larger than $r_{\mathrm{r}}$, no such geodesics are seen. They then occupy a crescent-shaped zone in between patches showing regions 1 and -7 (assuming the observer lies in region 1) at least till $r=r_{\text {pol }}$ (purple zone). Then the crescent becomes a thick shell separating regions 1 and -7 and, after outer horizon crossing, regions 1 and 3 (dark yellow zone). After inner horizon crossing, several possibilities arises, allowing the bounded region to take the shapes of a bubble, because region 3 is no longer visible (dark blue zone), or, alternatively, a secondary bubble within region 3 (gray zone). In any case, the bounded geodesic cease to be visible for sufficiently small $r$, unless the observer lies on the equatorial plane. The figure is made for $a=0.95 \mathrm{M}$. Other values of $a$ give the same structure (as long as $a^{2}<M^{2}$, of course), however some zones quickly become hard to see even for moderately smaller values of $|a|$. The two horizons are indicated for convenience as dark Grey vertical lines.

the bounded geodesics, i.e., null geodesics which admit a double real root at their largest root of $R(r)=0$, there is one whose turning point lies exactly at $s(e)=r_{-}$. Although it might seem unexpected since one may think that a geodesic crossing the inner horizon takes some time to bounce back from a negative $\dot{r}$ to a positive $\dot{r}$, visualization of the Carter-Penrose diagram (see Figure 7 ) tells that is is likely to happen in case some geodesic never enters the inner horizon region, i.e. regions 5 and $6(\bmod 8$, of course), which amounts to say that the geodesic passes directly from the ingoing inter-horizon region 2 to the outdoing inter-horizon region 12 . This happens if $R(r)$ admits $r=r_{-}$as a turning point. Given the form of $R(r)$, which includes a term proportional to $\Delta(r)$ (see Eq. (17)), this shall happen as soon as the remaining term, $\left(r^{2}+a^{2}\right) E-a L_{z}$ cancels out at $r=r_{-}$, which happens for $\xi=L_{z} / E=\left(r_{-}^{2}+a^{2}\right) / a=2 M r_{-} / a$. There are many such null geodesics since 
fixing $\xi$ is not enough, as $\eta$ is kept arbitrary. Stating that there exists a bounded null trajectory whose apoastron is $e_{-}$and "periastron" (notwithstanding the fact that this periastron occurs within the object of study) is $s\left(e_{-}\right)=r_{-}$is equivalent to solving Eq. (73) for this value, which yields (using the fact that we have of course $\left.a^{2}=2 M r_{-}-r_{-}^{2}\right)$,

$$
e_{-}^{4}-2 e_{-}^{3}\left(3 M-r_{-}\right)+e_{-}^{2}\left(r_{-}^{2}-4 M r_{-}+9 M^{2}\right)+2 e_{-} M\left(r_{-}^{2}-3 M r_{-}\right)+r_{-}^{2} M^{2}=0 .
$$

Setting $f=e_{-}-\left(3 M-r_{-}\right) / 2$ then leads to a significant simplification of the odd terms in powers of $f$ leading to a biquadratic equation:

$f^{4}-\frac{1}{2}\left(r_{-}^{2}-10 M r_{-}+9 M^{2}\right) f^{2}+\frac{1}{16}\left(81 M^{4}-180 M^{3} r_{-}+118 M^{2} r_{-}^{2}-20 M r_{-}^{3}+r_{-}^{4}\right)=0$.

The discriminant of this equation happens to be 0 , so that the solution is

$$
f^{2}=\frac{1}{4}\left(9 M^{2}-10 M r_{-}+r_{-}^{2}\right)
$$

Among the two solutions in term of $e$, we must keep those such that $e_{-}>r_{\mathrm{p}}$ which necessitates to keep the positive value of $f$, so that in the end, we have

$$
e_{s(e)=r_{-}}=\frac{3 M-r_{-}+\sqrt{\left(9 M-r_{-}\right)\left(M-r_{-}\right)}}{2} .
$$

(The same reasoning does not apply to $r_{+}$because although the above derivation remains valid, the corresponding value of $f^{2}$ is now negative.) Knowing this value of $e_{-}$and already knowing the value of $\xi$, we can deduce the values of $\eta$ through Eq. $(63)$ or any other equation involving $e$ and $\eta$ and $\xi$ and then deduce through Eq. 18 by imposing $\dot{\theta}=0$ the corresponding value of $\mu$. We did not find any simple expression for this, however.

\section{Cartesian Kerr-Schild coordinates}

\subsection{Why these coordinates are needed}

The last bit of implementation we need in order to simulate view deals with geodesics passing though the ring singularity, whether they are of transit or adventurous type. These geodesics are not mere academic features. There always within reach of an observer unless it is situated in the ingoing inter-horizon region $(2 \bmod 8)$. Unfortunately, the Kerr-Schild coordinates are not only singular along the polar axis (as any spherical - or, here, spheroidal - coordinates), but also at $r=0$. The reason is that it is now the $\theta$ coordinate that is not regular.

This can be seen by switching from the $\theta$ coordinate to the pseudo Cartesian coordinate $z:=r \cos \theta$. Passing through the ring singularity means that if we are in, says, region 5 with positive $z$, we shall end in region 7 with negative $z$, just as if we went though a $z=0$ plane in Cartesian coordinates. But in region $7, r$ is negative, so that both $z$ and $r$ change sign in the process. Consequently, if $z$ varies linearly 
in the process, $\cos \theta$ does not since it does not change sign. In other words, $\theta$ varies like

$$
\theta(p) \sim \frac{\pi}{2}+Z\left|p-p_{0}\right|,
$$

where $Z$ is some constant and $p_{0}$ is the value of affine parameter $p$ at ring singularity crossing. Consequently, although $\theta(p)$ is continuous at this event, $\dot{\theta}$ is not, so that Boyer-Lindquist coordinates cannot be used there.

This has to be overcome by switching to Cartesian-like coordinates $(r, \theta, \tilde{\varphi}) \rightarrow$ $(x, y, z)$. The fact that when $M=0$, the $g_{\tilde{\varphi} \tilde{\varphi}}$ coefficient is of the form $\left(r^{2}+a^{2}\right) \sin ^{2} \theta$ suggests that an good definition of $x$ and $y$ is such that $x^{2}+y^{2}=\left(r^{2}+a^{2}\right) \sin ^{2} \theta$. It then happens that a definition using complex numbers is more convenient, although is does depend on the choice of $\epsilon$. After some tries, one obtains that assuming

$$
\begin{aligned}
x+i y & =(r+i \eta a) \operatorname{sexp}(i \tilde{\eta} \tilde{\varphi}), \\
z & =r \mathrm{c},
\end{aligned}
$$

and assuming that the two quantities $\eta, \tilde{\eta}$ are both equal to \pm 1 , the new coordinate system is simply a Cartesian one in the case $M=0$ if the following constraint is satisfied:

$$
\eta \tilde{\eta}=\epsilon
$$

In what follows, we shall shall keep the usual orientation convention (despite $r$ being negative) and therefore impose that $\tilde{\eta}=1$, so that $\eta=\epsilon$.

When performing this transform, four-velocity/wavevector components also have to be changed. This is done using the Jacobian whose components are very easy to compute. One has for example

$$
V^{x}=\frac{\partial x}{\partial r^{\mu}} V^{\mu}
$$

where $V^{\mu}$ are the three spatial components expressed in Kerr-Schild spherical coordinates $r^{\mu}=(r, \theta, \varphi)$. The inverse transform is then obtained by inverting this matrix, which does not seem to possess a simple form ${ }^{f}$. One extra difficulty arises from the fact that even if we do not perform any coordinate change, we must keep track of the value of $r$. However, the relation between $r$ and the pseudo Cartesian coordinates $x, y, z$ involve only even powers of each coordinates:

$$
r^{4}-r^{2}\left(x^{2}+y^{2}+z^{2}-a^{2}\right)-a^{2} z^{2}=0 .
$$

This means that when we compute $r$ back from the $(x, y, z)$, we must keep track on which was the previous value of $r$ and whether on has passed though the ring singularity. The way this will be done in the case of null geodesics we are considering will be explained later, after we explain the properties of null geodesics that are of interest here.

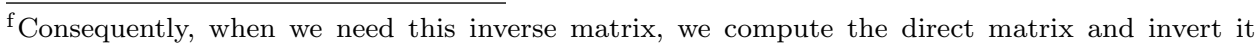
numerically. 


\subsection{New equations of motions}

Having defined the Cartesian Kerr-Schild coordinates, we now need to find the equations of motions in such coordinate system. The coordinate that most easily allows to compute its equation of motion is $z$. After short manipulations, we find

$$
\frac{\Sigma^{2}}{\mathrm{c}} \ddot{z}=\frac{R^{\prime}}{2}-\frac{2 r}{\Sigma} R-r \frac{\Theta^{\prime} \mathrm{s}}{2} \frac{\mathrm{c}}{\mathrm{c}}-r \Theta\left(1+\frac{2 a^{2} \mathrm{~s}^{2}}{\Sigma}\right)
$$

This equation of motion can further be expressed into a variety of ways, depending on whether one chooses to group first derivatives of the coordinates into constants of motion. Keeping in mind that in the case where $M=0$, the metric reduces to Cartesian coordinates, we expect that the equation of motion can be cast such that all terms in the right-hand side are proportional to $M$, just as this would be the case in a Newtonian framework and as it was also shown to be the case for the Schwarzschild metric 19. In this case, the next, most straightforward, step of the derivation is to expand the above expression by using Eqns. $\left[\begin{array}{ll|l|l|}17 & 18 & 21\end{array}\right.$.

All the term including powers of $E$ and $L_{z}$ cancel away, and so do terms proportional of $C$ and $\kappa$, except those among these that are proportional to $M$. We then obtain

$$
\Sigma^{2} \ddot{z}=-M z\left[\kappa r\left(\frac{4 r^{2}}{\Sigma}-3\right)+\frac{C}{r}\left(\frac{4 r^{2}}{\Sigma}-1\right)\right]
$$

The equation of motion for $x$ and $y$ is more difficult to obtain. Starting from the definition $(89)$, and performing two derivative with respect to proper time/affine parameter, we obtain straightforwardly

$$
\begin{aligned}
\Sigma^{2}(\ddot{x}+i \ddot{y})= & 4 i M r a W(\dot{x}+i \dot{y}) \\
& +\frac{x+i y}{r^{2}+a^{2}}\left[\left(r^{2}+a^{2}\right) F+r A+a B+i \epsilon\left(\left(r^{2}+a^{2}\right) D+r B-a A\right)\right],
\end{aligned}
$$

where the quantities $A, B, F$ and $D$ are defined by

$$
\begin{aligned}
A & =\frac{R^{\prime}}{2}-\frac{2 r}{\Sigma} R \\
B & =2 \Sigma^{2} \dot{\tilde{\varphi}} \epsilon \dot{r}-4 M r a W \epsilon \dot{r} \\
F & =c^{2} \frac{\Theta^{\prime}}{2 \mathrm{cs}}+\left(1-\frac{2 r^{2}}{\Sigma}\right) \Theta-\Sigma^{2} \dot{\tilde{\varphi}}^{2}+4 M r a W \dot{\tilde{\varphi}}, \\
D & =a X-2 \Sigma \dot{\tilde{\varphi}} r \dot{r} .
\end{aligned}
$$


The next step is to expand these four quantities, which transform into

$$
\begin{aligned}
A= & \frac{2 r}{\Sigma}\left(\left(r^{2}+a^{2}\right) E-a L_{z}\right)\left(a L_{z}-a^{2} \mathrm{~s}^{2} E\right) \\
& +r\left[\frac{2\left(r^{2}+a^{2}\right)}{\Sigma}-1\right]\left(C+\kappa r^{2}\right)-r\left(r^{2}+a^{2}\right) \kappa \\
& -M C\left(\frac{4 r^{2}}{\Sigma}-1\right)-M \kappa r^{2}\left(\frac{4 r^{2}}{\Sigma}-3\right) \\
B= & 2 \Sigma^{2} \dot{\tilde{\varphi}} \epsilon \dot{r}-4 M r a W \epsilon \dot{r}, \\
F= & \left(\frac{2 r^{2} \mathrm{~s}^{2}}{\Sigma}-1\right)\left(a E-\frac{L_{z}}{\mathrm{~s}^{2}}\right)^{2}-\mathrm{c}^{2}\left(a E-\frac{L_{z}}{\mathrm{~s}^{2}}\right) 2 \frac{L_{z}}{\mathrm{~s}^{2}} \\
& +\left(1-\frac{2 r^{2}}{\Sigma}\right)\left(C+\kappa r^{2}\right)+r^{2} \kappa \\
D= & a r W^{2}-2 a r W \epsilon \dot{r}+2 r a^{2} \mathrm{~s}^{2} W \dot{\tilde{\varphi}}-2 \Sigma r \epsilon \dot{\dot{r}} \dot{\tilde{\varphi}}-a r \kappa-a M W^{2}\left(\frac{4 r^{2}}{\Sigma}-1\right)
\end{aligned}
$$

Grouping these terms as in Eq. 96 then gives

$$
\begin{aligned}
\left(r^{2}+a^{2}\right) F+r A+a B= & -\left(\frac{4 r^{2}}{\Sigma}-1\right) M r C-\left(\frac{4 r^{2}}{\Sigma}-3\right) M r \kappa r^{2} \\
& -4 M r a^{2} W\left(\epsilon \dot{r}-a \mathrm{~s}^{2} \dot{\tilde{\varphi}}\right)+2 M r a^{2} W^{2} \\
\left(r^{2}+a^{2}\right) D+r B-a A= & \left(\frac{4 r^{2}}{\Sigma}-1\right) M a\left(C-a^{2} W^{2}\right) \\
& +\left(\frac{4 r^{2}}{\Sigma}-3\right) M a\left(\kappa r^{2}-r^{2} W^{2}\right) \\
& -4 M r^{2} a W\left(\epsilon \dot{r}-a \mathrm{~s}^{2} \dot{\tilde{\varphi}}\right) .
\end{aligned}
$$

A large number of terms appears in both expression, and it is easy to group them according to

$$
\begin{aligned}
& \left(r^{2}+a^{2}\right) F+r A+a B+i \epsilon\left(\left(r^{2}+a^{2}\right) D+r B-a A\right)= \\
& \quad-\left(\frac{4 r^{2}}{\Sigma}-1\right) M\left(C-a^{2} W^{2}\right)(r-i \epsilon a) \\
& -\left(\frac{4 r^{2}}{\Sigma}-3\right) M r\left(\kappa r+i \epsilon a W^{2}\right)(r-i \epsilon a) \\
& \quad-i 4 M r a W(r-i \epsilon a)\left(\dot{r}-\epsilon a \mathrm{~s}^{2} \dot{\tilde{\varphi}}\right) .
\end{aligned}
$$

This being done, one obtain a rather compact equation of motion for $x$ and $y$ :

$$
\begin{aligned}
\Sigma^{2}(\ddot{x}+i \ddot{y})= & 4 i M r a W\left(\dot{x}+i \dot{y}-\frac{x+i y}{r+i \epsilon a}\left(\dot{r}-\epsilon a \mathrm{~s}^{2} \dot{\tilde{\varphi}}\right)\right) \\
& -M \frac{x+i y}{r+i \epsilon a}\left[\left(\frac{4 r^{2}}{\Sigma}-1\right)\left(C-a^{2} W^{2}\right)+\left(\frac{4 r^{2}}{\Sigma}-3\right) r\left(\kappa r+i \epsilon a W^{2}\right)\right] .
\end{aligned}
$$

A last rearrangement further allows to find a more similar-looking form as compared to the $z$ equation by transforming the last $W^{2}$ into a $\kappa$ and by putting the 
corresponding difference in the first line. The whole, final, set of equations then reads:

$$
\begin{aligned}
\ddot{x}+i \ddot{y}= & \left.4 i M a \frac{r}{\Sigma^{2}} W\left[\dot{x}+i \dot{y}-\frac{x+i y}{r+i \epsilon a}\left\{\dot{r}-\epsilon a \mathrm{~s}^{2} \dot{\tilde{\varphi}}+\left(\frac{4 r^{2}}{\Sigma}-3\right) \epsilon \frac{W^{2}-\kappa}{4 W}\right\}\right] 09\right) \\
& -M(x+i y) \frac{r}{\Sigma^{2}}\left[\left(\frac{4 r^{2}}{\Sigma}-3\right) \kappa+\left(\frac{4 r^{2}}{\Sigma}-1\right) \frac{C-a^{2} W^{2}}{r(r+i \epsilon a)}\right] \\
\ddot{z}= & -M z \frac{r}{\Sigma^{2}}\left[\left(\frac{4 r^{2}}{\Sigma}-3\right) \kappa+\left(\frac{4 r^{2}}{\Sigma}-1\right) \frac{C}{r^{2}}\right] .
\end{aligned}
$$

As a first crosscheck, we can notice that when setting $a=0$, these equations reduce to those that are valid for the Schwarzschild metric (see Ref. 19). The term proportional to $\kappa$ corresponds to the Newtonian term and after performing the substitution $C \rightarrow L^{2}$, where $L^{2}$ is the particle total angular momentum (or angular momentum per unit of mass for timelike geodesics), this term corresponds to the Schwarzschild term. Moreover, expanding this expression in powers of $a$ allows to recover the gravitomagnetic term for non relativistic particles. We give some details about this in Appendix A.

We can now go back to the problem of determining how to handle ring singularity crossing. This is done as follows. Firstly, we care about this issue only for geodesics that have been identified as crossing the ring singularity, see 4.6 . Secondly, it is known that null geodesics either oscillate around the equatorial plane or exist in a limited interval of $\theta$ which is comprised somewhere within $] 0, \pi / 2[$ (or within $] \pi / 2, \pi\left[\right.$ ). Thirdly, it has been shown $\frac{12}{12}$ that null geodesics crossing the ring singularity only belong to this latter category. Consequently, geodesics crossing the ring singularity will experience simultaneously a sign flip for both $z$ and $r$, and, according to the third point above, the flip in $z$ occurs only once, at ring singularity crossing. Therefore, the evolution of the $z$ quantity suffices to detect the ring singularity crossing at which the sign of $r$ must be flipped when solving Eq. (93).

Incidentally, this means that there are not one set of Kerr-Schild coordinates, but four. First because we must choose among the two values of $\epsilon$ (although the choice of value does not matter at ring singularity crossing), and second we must consider the choice with the product $z r$ being either positive or negative. The choice $z r>0$ is suitable when we go from the $z, r>0$ region to the $z, r<0$ one, that is we enter into the negative $r$ region by diving into the ring singularity from above. This same coordinate system (or these same coordinate systems, since there are two such systems depending on the value of $\epsilon$ ) can also be used when going back to the $r>0$ side by springing upward from the ring singularity. The two other sets of Kerr-Schild coordinates (with $z r<0$ whichever value of $\epsilon$ ) must be used for the two other crossings (going into the negative $r$ region from below the equatorial plane, etc.).

In the problem of horizon/ring singularity crossings, the last but crucial step is to find a procedure for the choice of the affine parameter so that the jumps in $r$ at each integration step are not too large so that we have time to switch from one 
coordinate system to another when necessary. For example, if we are in region 1 and integrate backward a geodesic coming from the outer horizon, then this geodesic originates further back from the inner horizon. If the value of $\epsilon$ must be changed when crossing these two horizons, then our step of integration must send $r$ first in the inter-horizon region ( 4 in this case) before switching to the other set of KerrSchild coordinate an continue the integration for the inner horizon crossing. This is done by finding upper bounds on the possible variations of $r$ as a function of the affine parameter by fiddling with Eq. (17). We then use standard numerical rounites 22 to solve the geodesic equations.

\section{Simulated views, 2nd part}

We now come to the graphical illustrations of the previous discussions of 4 Details of our raytracing software has been given elsewhere ${ }^{14}$, so that we shall only very briefly summarize the features that are of interest here.

Since in many situations we need to observe our scenery along very different directions (in term of angular distance), we need to compute simulation on a very large field of view. The most convenient projection we found for this purpose is the azimuthal equidistant projection, which is now commonly used in any digital planetarium under the nickname of DomeMaster formatg

Our simulations take into account aberration due to observer's motion and can also implement Doppler effect. They naturally take into account lensing of extended objects by showing their pixel by pixel distortion. We can also compute lensing of individual stars, i.e. magnification. However, in order to focus on the aspects of visualization that have been described above, we chose to either strongly attenuate or even remove the Doppler shift from our simulated views, as those induced far too contrasted images. We also removed stars from our simulations, the main reason being that computing the distortion of a celestial sphere amounts to solve backward the geodesic equations from the observer position to some past null infinity, which can be done in one shot. Adding star necessitates to further perform direct raytracing, which is much more time-consuming. Moreover, some extreme lensing phenomena prevented from obtaining a satisfactory rendering.

The main issue we had to address was the distortion pattern of the different asymptotic regions our fiducial observer would see as a function of its position. Our first guess was to use simple coordinate grids for each asymptotic regions, but those happened to be too complicated to interpret in most contexts. Moreover, the different asymptotic regions were too difficult to distinguish from another in this case. Therefore, it was was mandatory to use several different celestial spheres:

- Observer's initial region, region 1 is covered by the starless Milky Way seen in the near infrared as observed by the Two-Micron All-Sky Survey 15 .

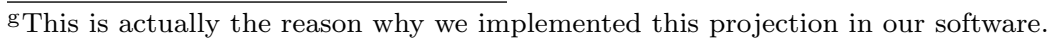


- Region 1 twin, i.e., region 3, used the Milky Way seen by Planck satellite High Frequency instrument (mostly dominated by the $857 \mathrm{GHz}$ map) after a one-year full-sky survey 23 .

- Negative $r$ regions 7 and 8 show CMB full-sky maps coming from Planck full mission CMB-only map 24 and WMAP 9 year Internal Linear Combination Map 25, respectively. We also use the same Planck map for region -1 whom a tiny portion is seen from region 1 . This cannot introduce any confusion since regions -1 and 7 cannot be seen simultaneously nor immediately one after the other.

- We made an exception regarding region -7 whose celestial sphere is a coordinate grid with $5^{\circ} \times 5^{\circ}$ grayscale patches (whose colors actually match some black body). Polar regions are orange, and we used different color for four meridians situated $90^{\circ}$ apart from each others (whitish, light gray, dark and dark red). We also used a different colors for the northern and southern equatorial bands (very light gray, and dark, respectively).

We first want to illustrate how different the wormhole silhouette can look as a function of the observer's latitude and velocity (Fig. 10p. Along a polar, infalling trajectory, the wormhole silhouette appears perfectly circular. Region -7 occupies almost all of the space within in but only if $r$ is sufficiently large. For smaller $r$, it is surrounded by the dark shell phenomenon described in the previous Section and which also occurs in the Reissner-Nordström case. However, contrarily to the latter, the Kerr case induces a rotational deformation of region -7 as can be seen by following the swirling patterns of the meridians. An almost polar trajectory from region -7 does not experience the Lense-Thirring effect, but so do trajectories with increasing impact parameter. Since we are here looking at a counterclockwise rotating wormhole from above, the swirls are also counterclockwise from center to edge (Fig. 10 top left). Note that in the middle of region -7 , a small patch of region -1 is also seen. When near the equator, the dark shell occurs, but before, the (infalling) observer will see the dark crescent phenomenon (Fig. 10, top right), which appear on the retrograde side of the wormhole. Region -1 is also seen but this time along a flattened ellipse since the singularity is seen much more edge-on. If we now consider the same observer but who is now outgoing (by flipping the $r$-component of its four-velocity), a huge aberration "unfolds" the whole wormhole silhouette which now occupies most of the celestial sphere, with an angular diameter significantly larger than $\pi$ (Fig. 10 bottom left). If one looks along the opposite direction of the wormhole (Fig. 10, bottom right), the current region the observer is in appears within a small patch of the sky. The dark crescent is still there, but it now looks like a sort of "outgrowth" protruding over region 1's patch.

After this warm-up, we now show a more detailed sequence seen by an observer travelling from region 1 to region 7 . We shall consider an observer freely-falling on the Kerr wormhole starting from a zero velocity and angular momentum at infinity, i.e., $E=1, L_{z}=0$. The Carter constant is chosen as $C=a^{2}$ so as $\theta$ to remain 

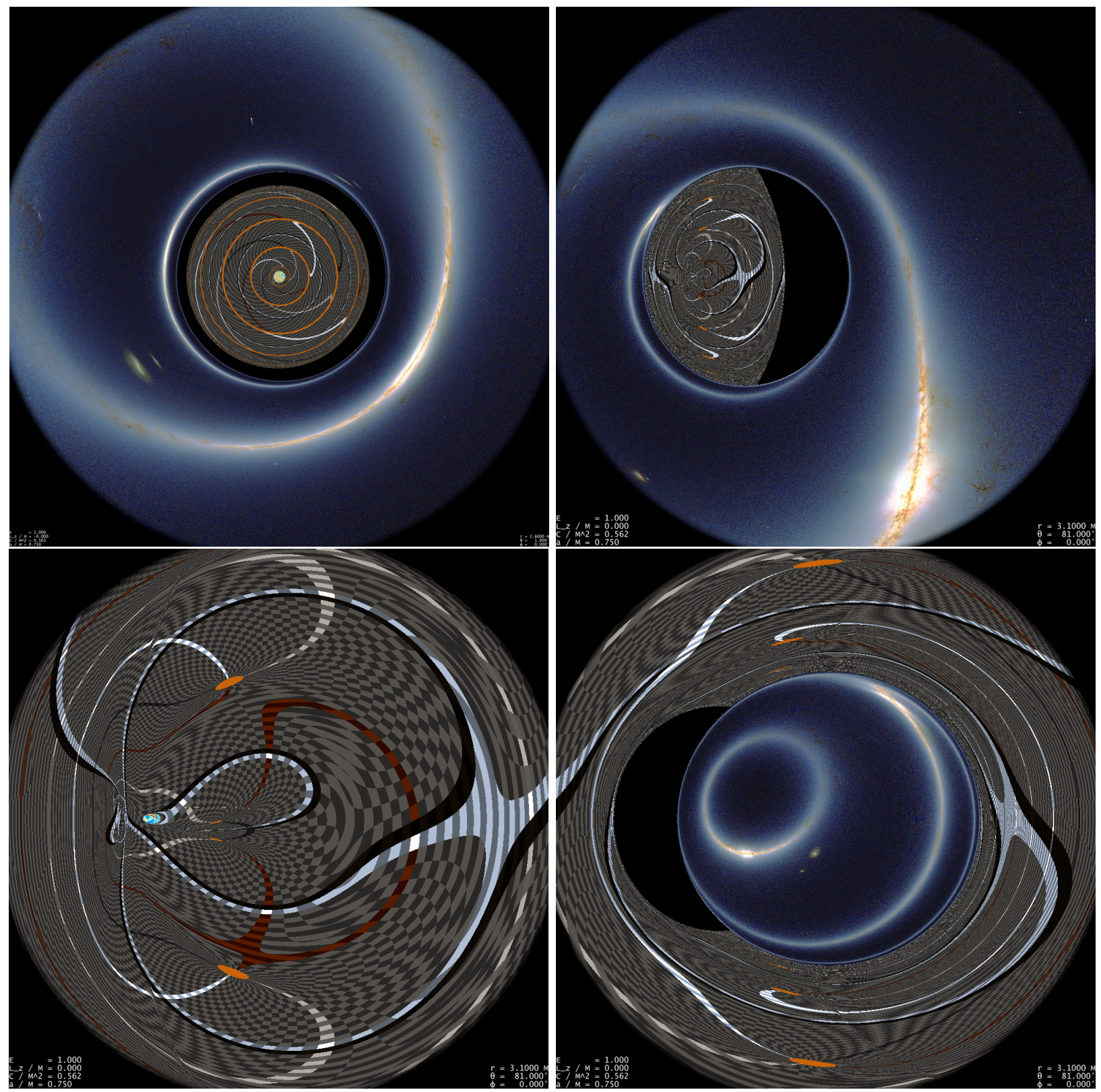

Fig. 10. Observer in three situations: (i) polar, infalling trajectory at $r=2.6 M$ (top left), close-toequatorial $\left(\theta=81^{\circ}\right)$ infalling at $r=3.1 M$ (top right) and close-to-equatorial $\left(\theta=81^{\circ}\right)$ outgoing at $r=3.1 M$ (bottom left). This view correspond to the same position as in the infalling one, except that we have flipped the sign of the observer's $\dot{r}$. The bottom left view shows the opposite direction of that of bottom right view. 
constant. If we want to see through the ring singularity, we cannot consider the case of an observer being on the equatorial plane, but a too large latitude will prevent us from seeing all the features outlined in Fig. 9. We therefore choose $\theta=81^{\circ}$ (i.e., northern latitude of $9^{\circ}$ ). With these constants of motion, the observer will penetrate into the wormhole, cross the two horizon and then bounce when reaching the ring singularity $\left(r=0, \theta=81^{\circ}\right)$. We then decide to push the observer in the negative $r$ region. This part of the journey will not be done along a geodesic. In order to do so we would need to start with a fairly high value of $E$, which would significantly shrink several zones of interest because of aberration. Therefore, the journey into the $r<0$ region will be shown as seen by a quasistatic observer. Even with all these requirements, it is not very easy to find a value of $a$ which allows to see well all the features related to the null geodesics. After several tries, we found that the value of $a=0.75 M$ was a good compromise. Also, the values of $\theta$ and $a$ allow to intersect all the regions of interest regarding the bounded geodesics of Fig. 9: the complete wormhole, then the dark crescent, the dark shell, the dark shell together with the secondary bubble, then the dark shell alone again, the dark bubble and then nothing, followed by the crossing of the ring singularity.

During the overall trajectory there are huge Doppler effects that are involved in many directions, thus making the images impossible to interpret. We therefore removed on purpose such Doppler shift in order to focus on the shape of the different patches of the sky that we can see. Lastly, as the observer is moving, its $\varphi$ coordinate (in the Boyer-Lindquist frame) varies a lot. However, we found that this variation led to more confusion than enlightenment as this drift in $\varphi$ led to large changes in the aspect of the various celestial spheres. Therefore we have (rather disputably, we admit) chosen not to follow the evolution of the $\varphi$ coordinate, despite computing the view with the correct value of $\dot{\varphi}$, i.e., the one imposed by the constants of motion we have chosen.

This being said, the next sets of images show what we think are the most interesting features of this numerical exploration.

The first set of images (Fig. 11) shows the progress toward the wormhole outer horizon at coordinate distance at $r=6 M, 3 M, 1.8276 M$ and $1.6781 M$. The first picture is well above the bounded geodesics regions. It therefore shown region 1 as well as, inside the wormhole silhouette, region -7 . Within this region, a very tiny bit (almost impossible to see) of region -1 where $r$ is negative. More will be seen later about negative $r$ region, so that it is not necessary to focus on it at this stage. The shape of the wormhole silhouette is slightly different than in Fig. 11, first because the observer is slightly off the equatorial plane and mostly because the black hole is far from extremal for this purpose (the black hole shape with a flattened prograde side occurs only when $a$ is very close to $M$. Inside the wormhole, we see a very distorted view of region -7 The colored poles as well as the four main meridian and the equator form somehow regular but highly distorted patterns. At $r=3 M$, the observer enters into the region where bounded geodesics are seen. The dark patch corresponding to those first appears on the retrograde side of the wormhole. 
This is what we had called the dark crescent. At $r=1.8276 M$, the dark crescent has spread over the whole wormhole edge. However since we are still outside the outer horizon, region -7 is still visible. At $r=1.6781 M$, the size of region -7 has drastically diminished. This is because the observer is very close to the wormhole outer horizon $\left(r_{+}=1.6614 M\right.$ with our value of $\left.a\right)$.

The next set of images (Fig. 12) shows the inter-horizon region. There, only region 1 and its mirror, region 3 , are seen. The snapshots we show are situated at $r=1.6448 M, M, 0.4 M$ and $0.3389 M$. Region 3 appears at the exact spot where region -7 had disappeared. As its angular size grows, we notice that more or less half of it appears reasonably undistorted (one recognizes the Milky Way band), whereas of the right (i.e., retrograde) part show a "whirlpool"-like pattern. The relative size of the highly distorted region as compared to the less distorted region increases at the observer gets closer and closer to the inner horizon, at $r_{-}=0.3386 \mathrm{M}$. At first, it seemed to us that such whirlpool-like pattern was a little bit unexpected as region 1 appears fairly undistorted in comparison. There are two reasons for this. Firstly, the celestial sphere of region 1 does not possess many features as opposed to region 3, which makes the comparison difficult. The second (and main) reason is more subtle. Once in region 2, regions 1 and 3 share very similar role (as opposed to Fig. 11 where region 1 and -7 when both were seen from region 1 ). The only difference between the two is that our observer has an $E$ equal to 1 whereas a similar observer coming from region 3 would have an opposite $E$ and otherwise identical constants of motion. In other words, passing from our observer to its mirror analogue just amount to perform a Lorentz boost. But it this Lorentz boost is made in the direction opposite to that where there is a large distortion, then the angular size of the patch will enormously increase, giving the impression that the amount of distortion increases. But this is merely an artefact of the zooming power of aberration induced by large Lorentz boost. We give a few figures about this in Appendix B. Figure 13 show the progressive distortion that occurs when performing a Lorentz boost in order to change the observer's $E$ from 1 to -1 . The symmetric case where $E=0$ (sometimes dubbed as "lazy geodesic" clearly shows that both regions 1 and 3 experience the same amount of distortion, although with our choice of celestial spheres the actual amount of distortion is more readily apparent in region 3 , the detail of which will deserve further scrutiny.

Continuing the free-fall within the inner horizon (Fig. 14) allows to see several changes in the topology of the bounded geodesics regions. At $r=0.3047$, i.e., very soon after inner horizon crossing, a tiny patch the the sky show the region 7 , with negative $r$ (upper left image of Fig. 14). This region appears at the edge of the bounded geodesics shell, although at horizon crossing, the shell very temporarily gets pinched in one point by the region 3 patch. A new type of distortion within the region 3 patch appears close to the pinch. Decreasing $r$ makes the patch of region 7 grow larger and also allows for the inner, secondary dark bubble of bounded geodesics to appear (upper right panel, $r=0.1 M$ ). The secondary bubble does not last long and has already disappeared at $r=0.02 M$. At this point, the patch of 

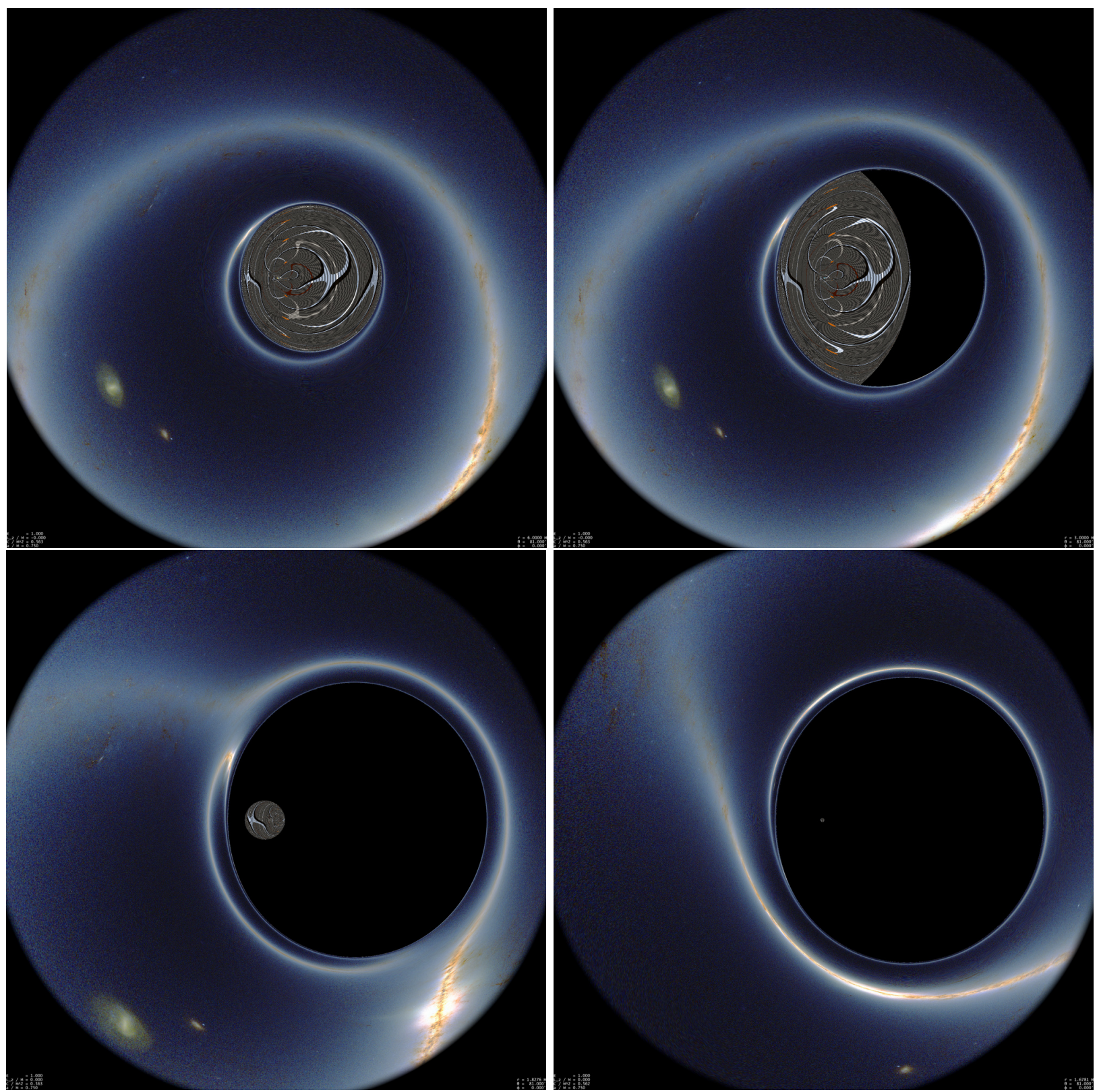

Fig. 11. Infalling observer at $r=6 M, 3 M, 1.8276 M$ and $1.6781 M$.

region 7 keeps on increasing in size, but that of region 3 (within the dark shell) decreases In this image, we have outlined the ring singularity by white pixels. As already explained, within the ring singularity we see both region 7 and some bits of region 1 through the adventurous geodesics which are traveling back from their 


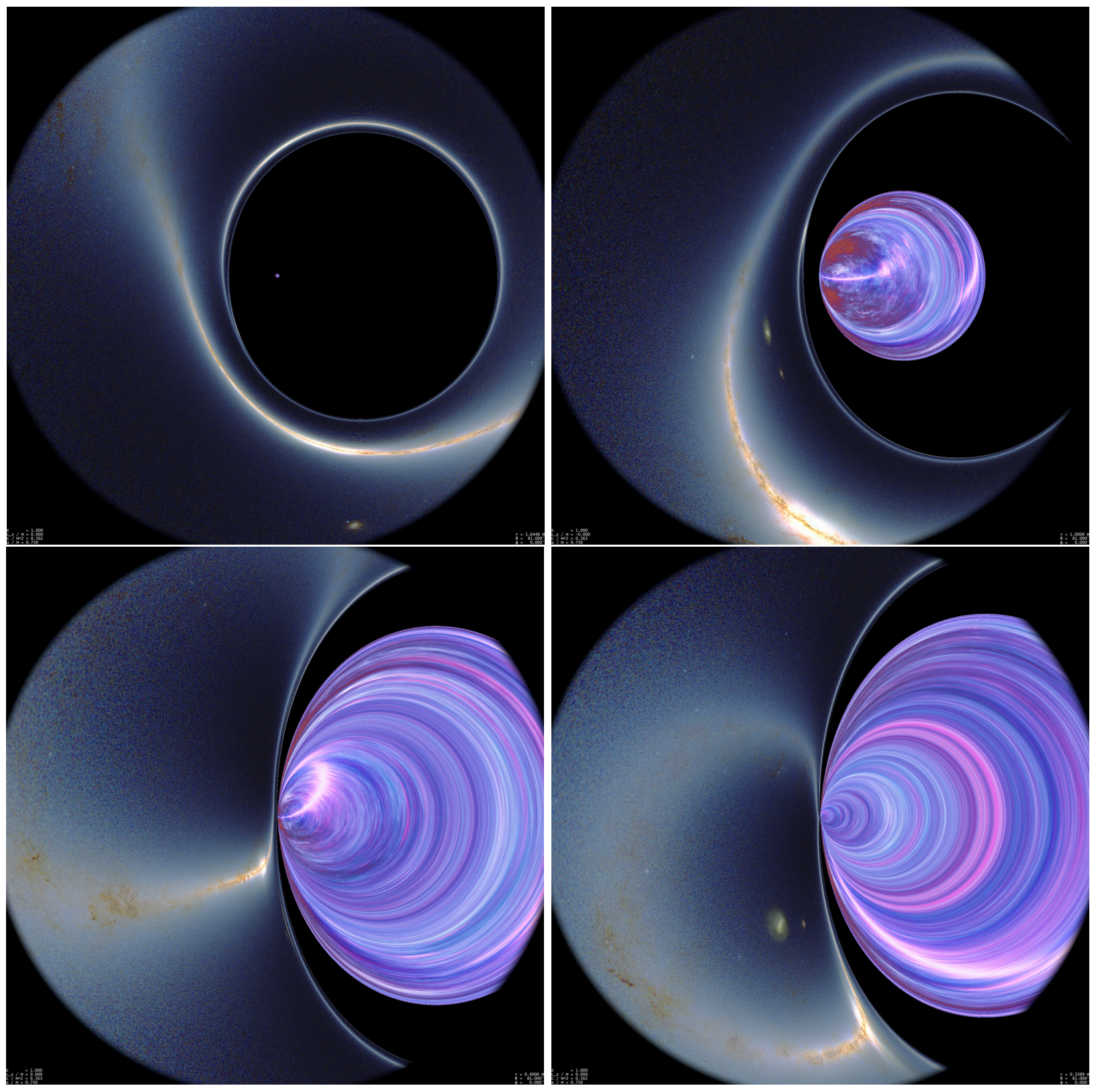

Fig. 12. Infalling observer at $r=1.6448 M, M, 0.4 M$ and $0.3389 M$.

short trip in the negative $r$ region. At some point $(r=0.120 M$, lower right image), the distortion of region 3 is completely dominated by the whirlpool-like pattern. Note at this point that since we are now within the inner horizon, regions 1 and 3 are no longer equivalent. The dissymmetry in their distortion patterns is no longer 

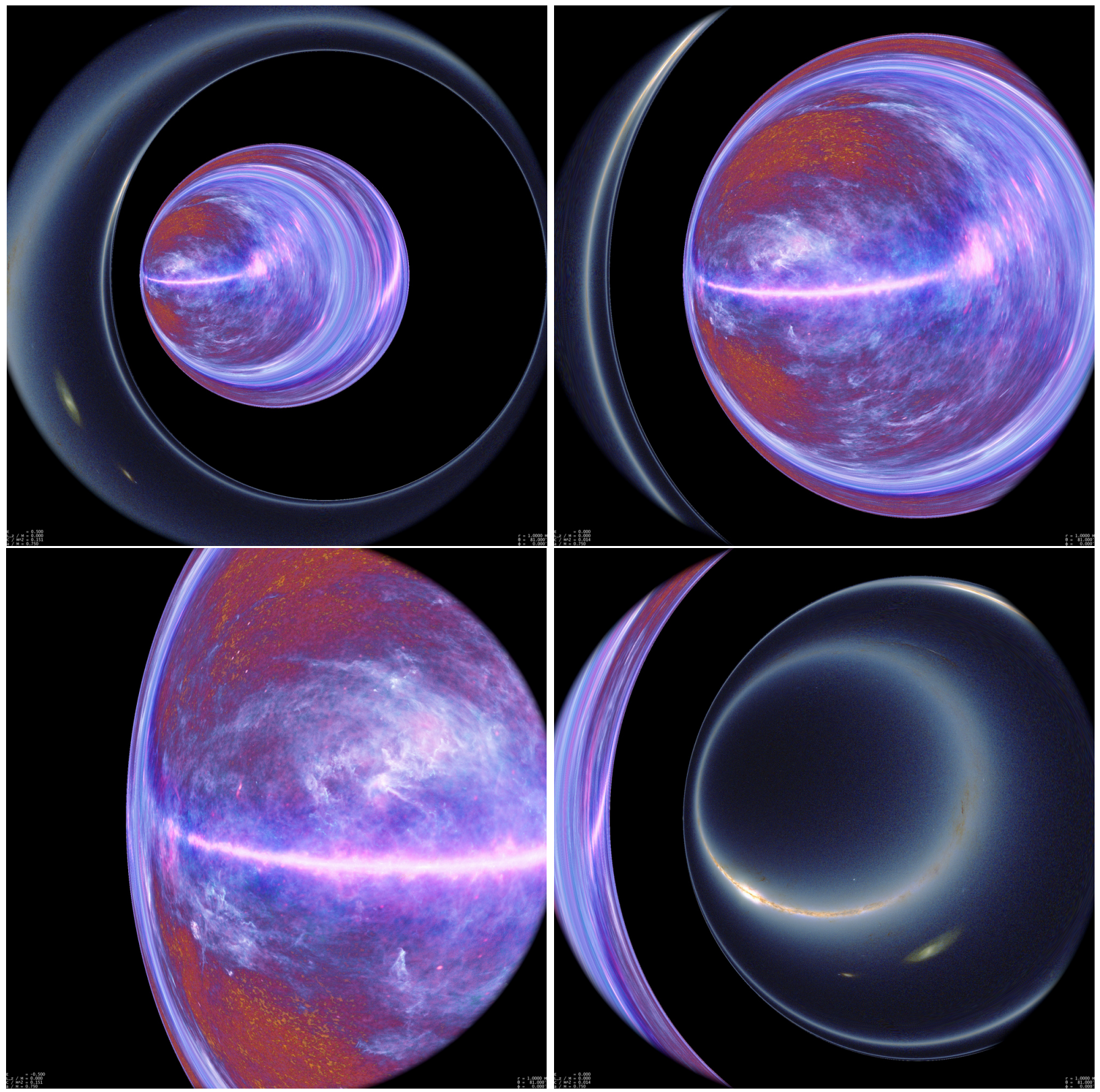

Fig. 13. Observer with varying $E$ at $r=M$. Starting from Fig. 12 upper right image, we reduce $E$ from to 0.5 (top left image), then 0 (top right) and -0.5 (bottom left). Bottom right image shows the opposite direction for $E=0$ and illustrates the perfectly symmetric role of the two regions.

an issue.

The next step of the journey consists in getting closer and closer to the ring 

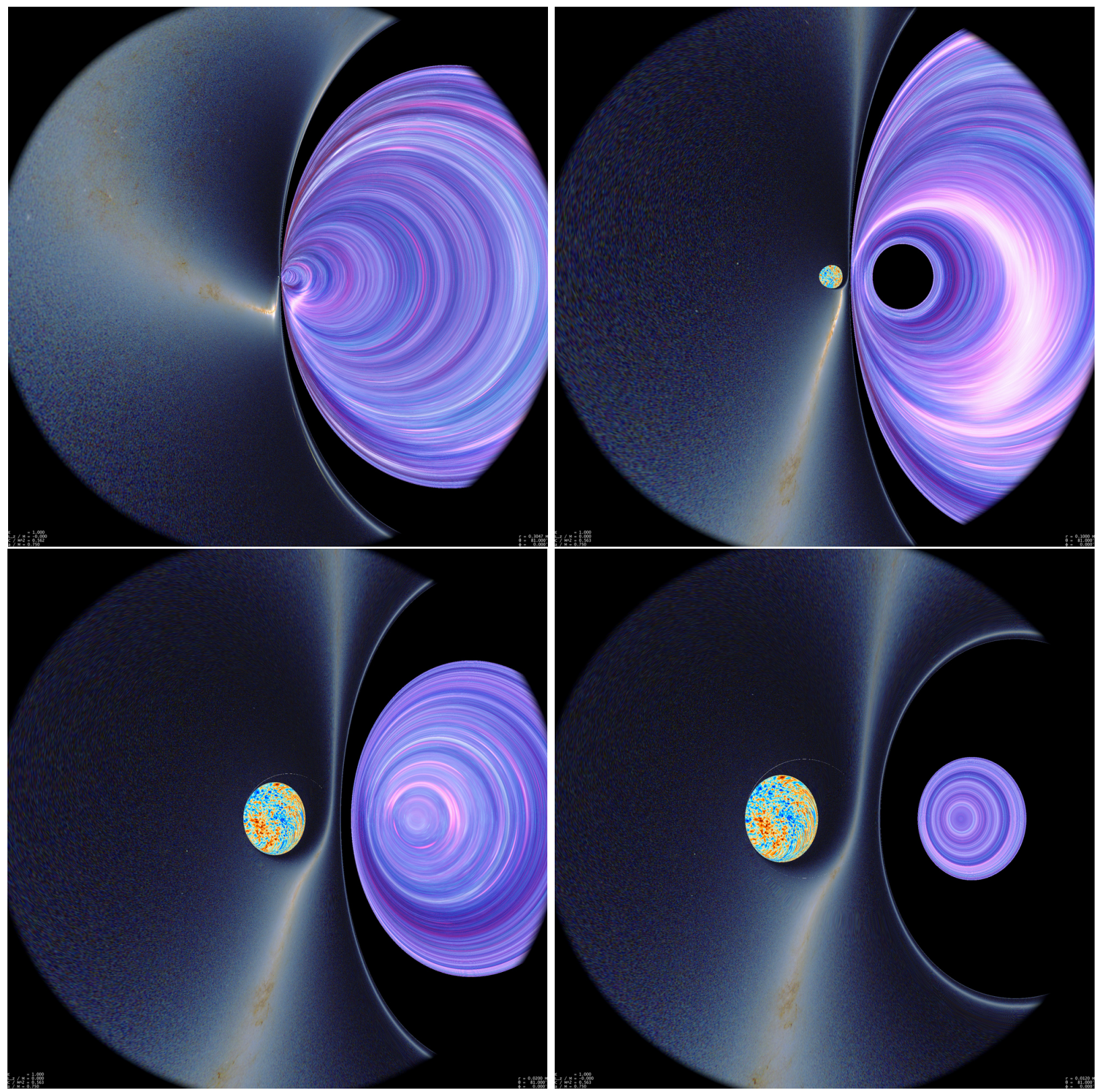

Fig. 14. Continuation of the free-fall, now within the inner horizon, at (from left to right and top to bottom) $r=0.3047 M, 0.1000 M, 0.0200 M$ and $0.0120 M$.

singularity. According to Fig. 9, the various steps are first the disappearance of region 3 at latest when the observer leaves the inner ergoregion (Fig. 14, top left image). At this point the "dark shell" transforms into a dark bubble which is also 
led to shrink (upper right image) and then disappear (bottom right image). We have found that a whirlpool-like pattern is briefly seen in the patch of region 1 in the direction where the dark bubble disappeared. From now on, only regions 1 and 7 are seen, the latter having a growing angular size (bottom right image). Two interesting features are worth mentioning at this stage. Firstly, we do not see the whole celestial sphere of region 7 . This was already the case before but it now becomes more obvious. The reason is that transit geodesics experience a limited amount of variation of their $\theta$. Conversely, at a given observer's colatitude $\theta$, there is only a small interval around this $\theta$ from which geodesics can originate from the celestial sphere. Since transit geodesics are also limited in term of their $\xi$ parameter ${ }^{12}$ the same remark is very likely to apply in term of longitude as well. The second obvious feature is that the ring singularity has a shape closer to that of a circle than an ellipse. This may look surprising since the observer's latitude is large, however, without even considering light deflection, it has to be noted that the ring singularity is visible only after inner horizon crossing, where the $r$ in KerrSchild coordinates is already small. Consequently, at least in term of coordinates, the observer is more "above" the singularity than close to its plane. This seems the most likely explanation of the visual roundness of the singularity. Let us add that this is not an artefact of aberration: aberration transforms circles into circles ${ }^{26}$ so that any observer situation at the same point but with different velocities would also see the singularity shaped close to a circle.

Crossing the ring singularity does not go along with significant changes in what the observer sees Fig. 16. This is not completely surprising since the actual "crossing" of the singularity cannot be associated with the crossing of some special hypersurface just as horizon crossing is. The fact that we have implicitly decided that singularity crossing occurs at $r=0$ is a rather disputable choice motivated by the seemingly naturalness of the Kerr-Schild coordinate system. Another possibly more relevant criteria would be the envelope of the adventurous geodesics which would include part of the negative $r$ region. In any case, when $r=0$, the ring singularity corresponds to a great circle from the point of view of our observer, which in this case is a static observer. Whether or not this result could be expected also deserves a further study. However, if we are interested in the angular size of the negative $r$ region, then it still occupies a limited patch in the sky: from the observer's point of view, most of the angular area what originates from the negative $r$ region corresponds to adventurous geodesics that are on their way back to the positive $r$ region.

Advancing into the negative $r$ region allows to see a larger and larger part of the negative $r$ region celestial sphere (Fig. 17). We find that it is soon before $r \sim-0.09 M$ that the negative $r$ region spread over more than half of the celestial sphere (bottom left image).

The view at $r=-0.5120 M$ is in our opinion the most aesthetic of this simulation (Fig. 18). This image shows a nice swirling pattern around what remains visible 


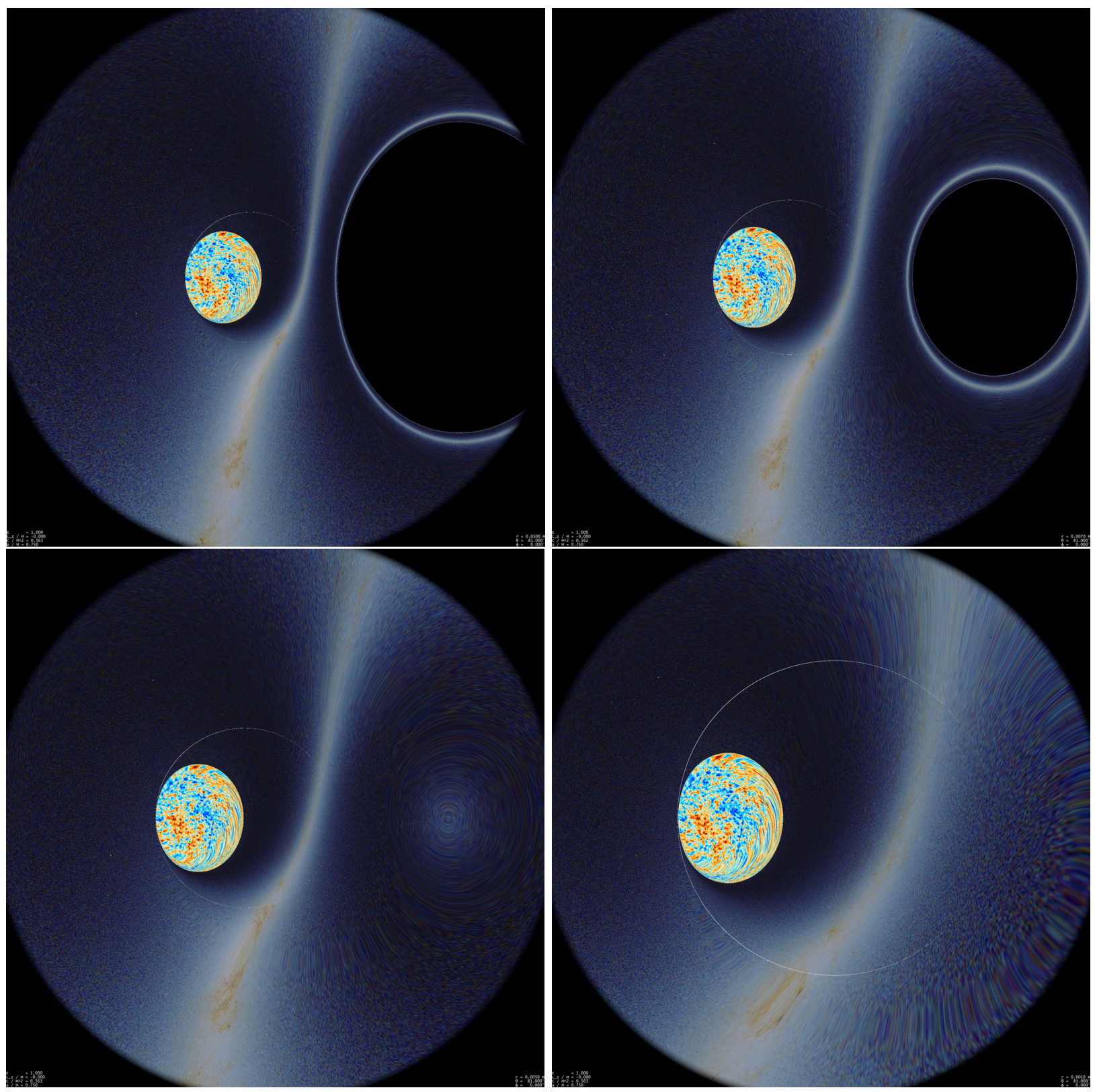

Fig. 15. Approaching the ring singularity at (from left to right and top to bottom) $r=0.0100 M$, $0.0070 M, 0.0050 M$ and $0.0010 M$.

from region 1. Such pattern is of course not unexpected since the metric deals with a rotating singularity, however, the apparent rotation of the swirl remains to be investigated as well. Another feature of interest is that we only see a limited part 

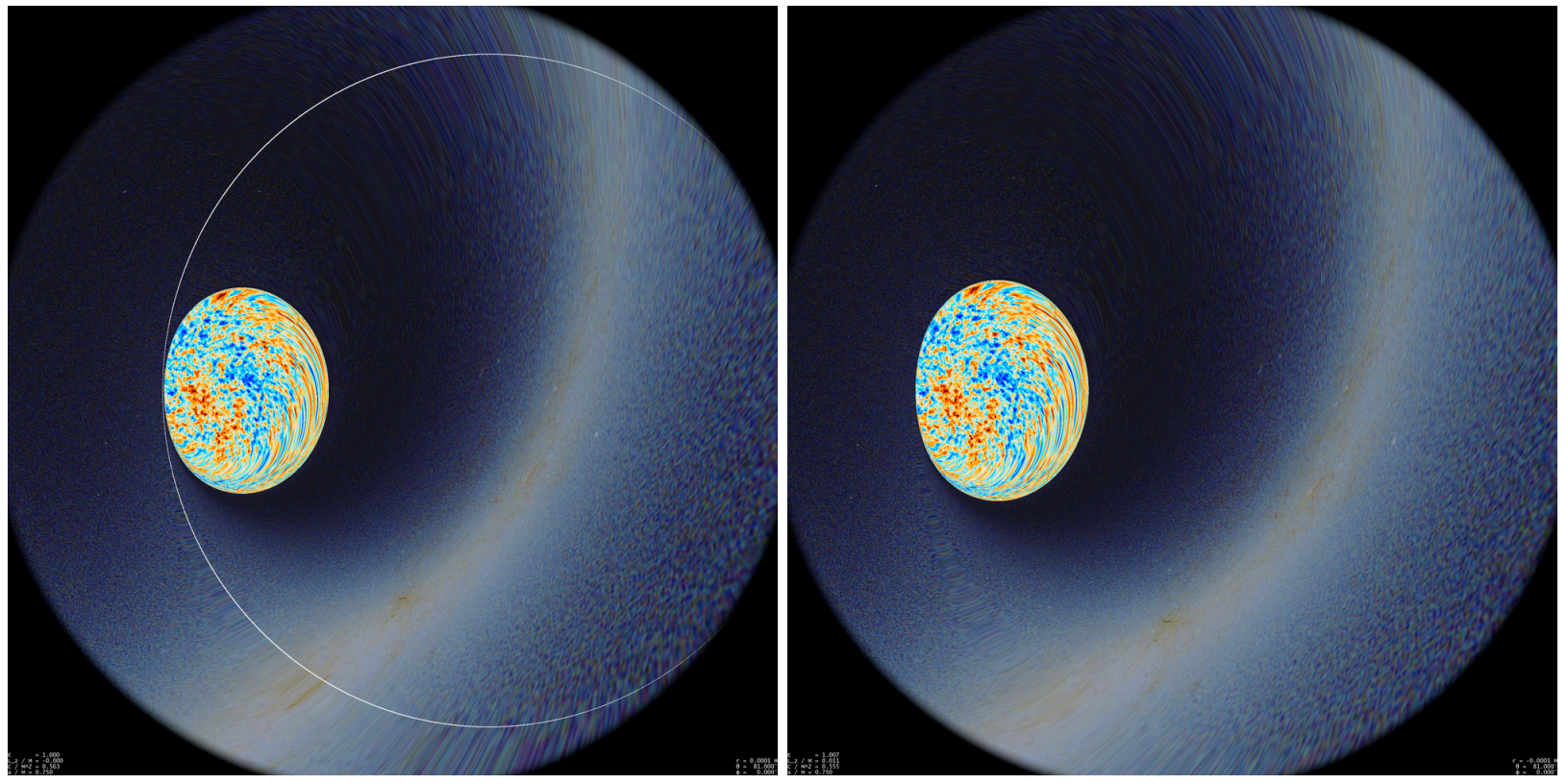

Fig. 16. Before and after crossing the ring singularity at $r=0.0001 M$ and $r=-0.0001 M$.
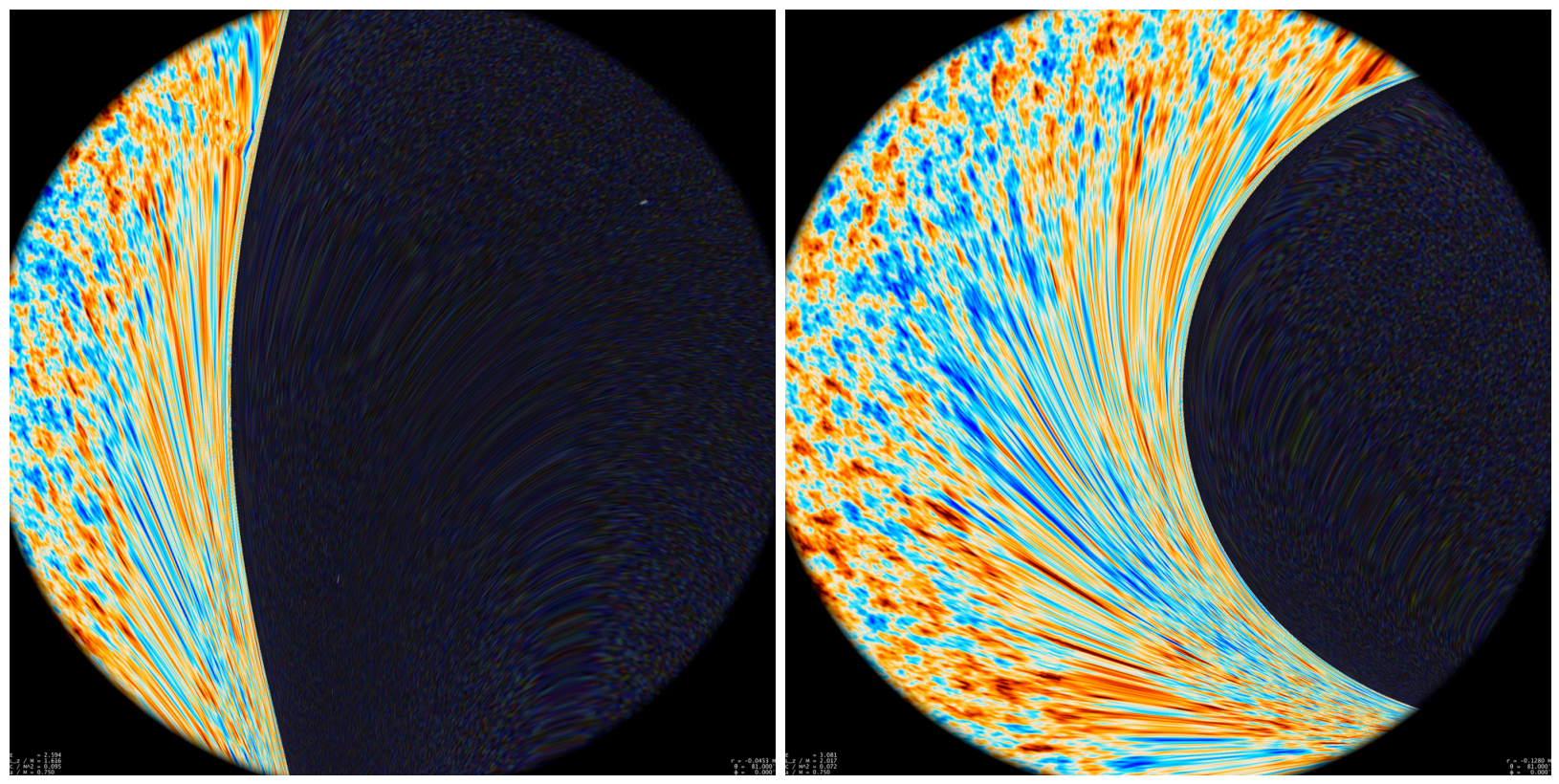

Fig. 17. Escaping toward the negative $r$ region along a quasi-static trajectory, at $-0.0453 M$ (left) and $-0.1810 M$ (right). 
of region 1 , the reason being the very same as the one which prevented from seeing the whole celestial sphere of region 7 from region 5 .

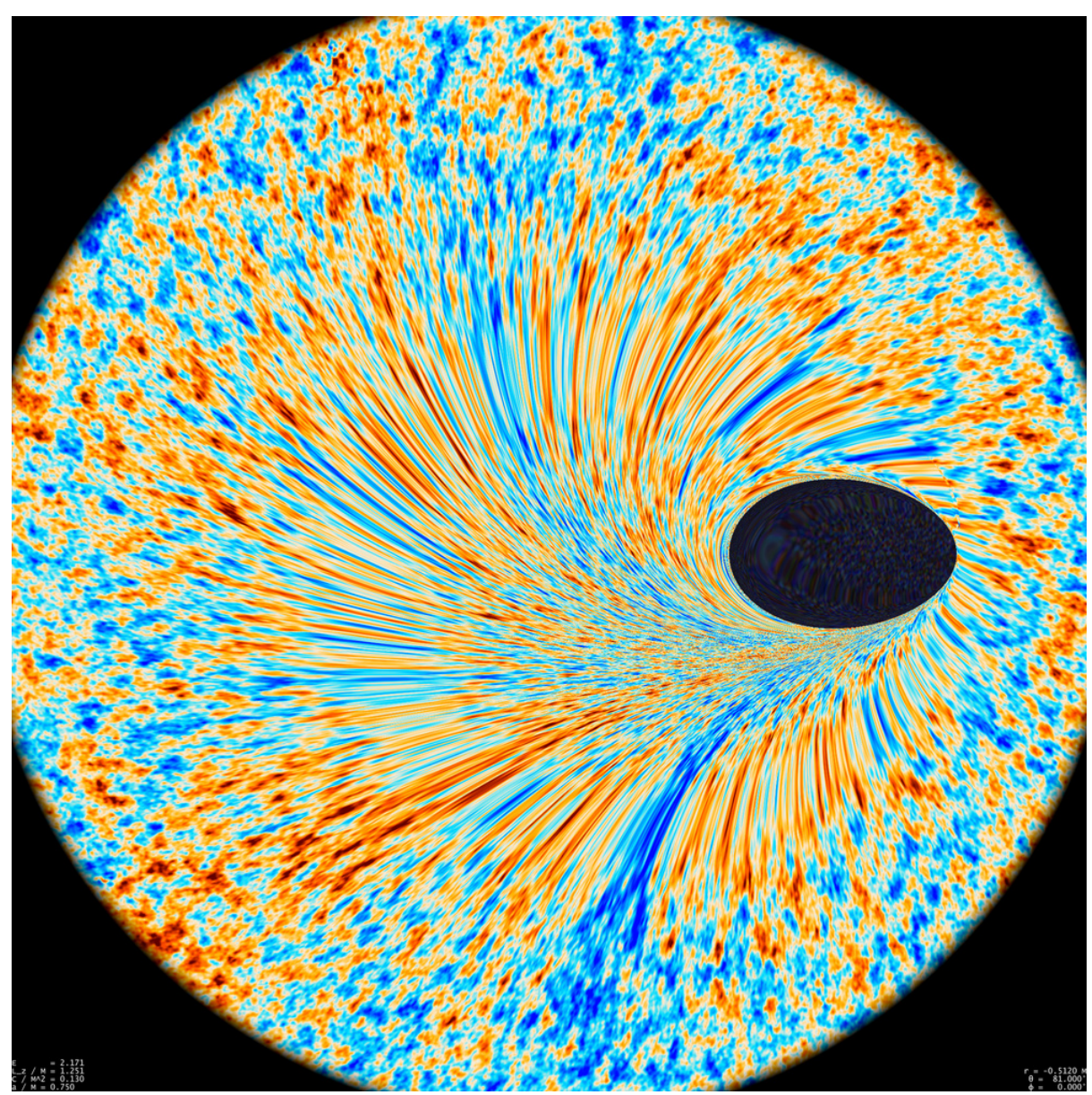

Fig. 18. A beautiful snapshot of the observer's region of origin seen from the negative $r$ region, $r=-0.5120 M$

Going even further within the negative $r$ region gives rise to phenomena which seem more easy to interpret Fig. 19. The celestial sphere of region 7 shows multiple images along directions that are close to the singularity as well as a radial shear distortion which are characteristic of negative mass systems.

\section{Conclusion}

In this paper, we have studied from a visual point of view some aspects of the Kerr metric, focusing on its maximal analytic extension, but also on the very viciniry of a realistic black hole. Although several features of the metric can be known qual- 

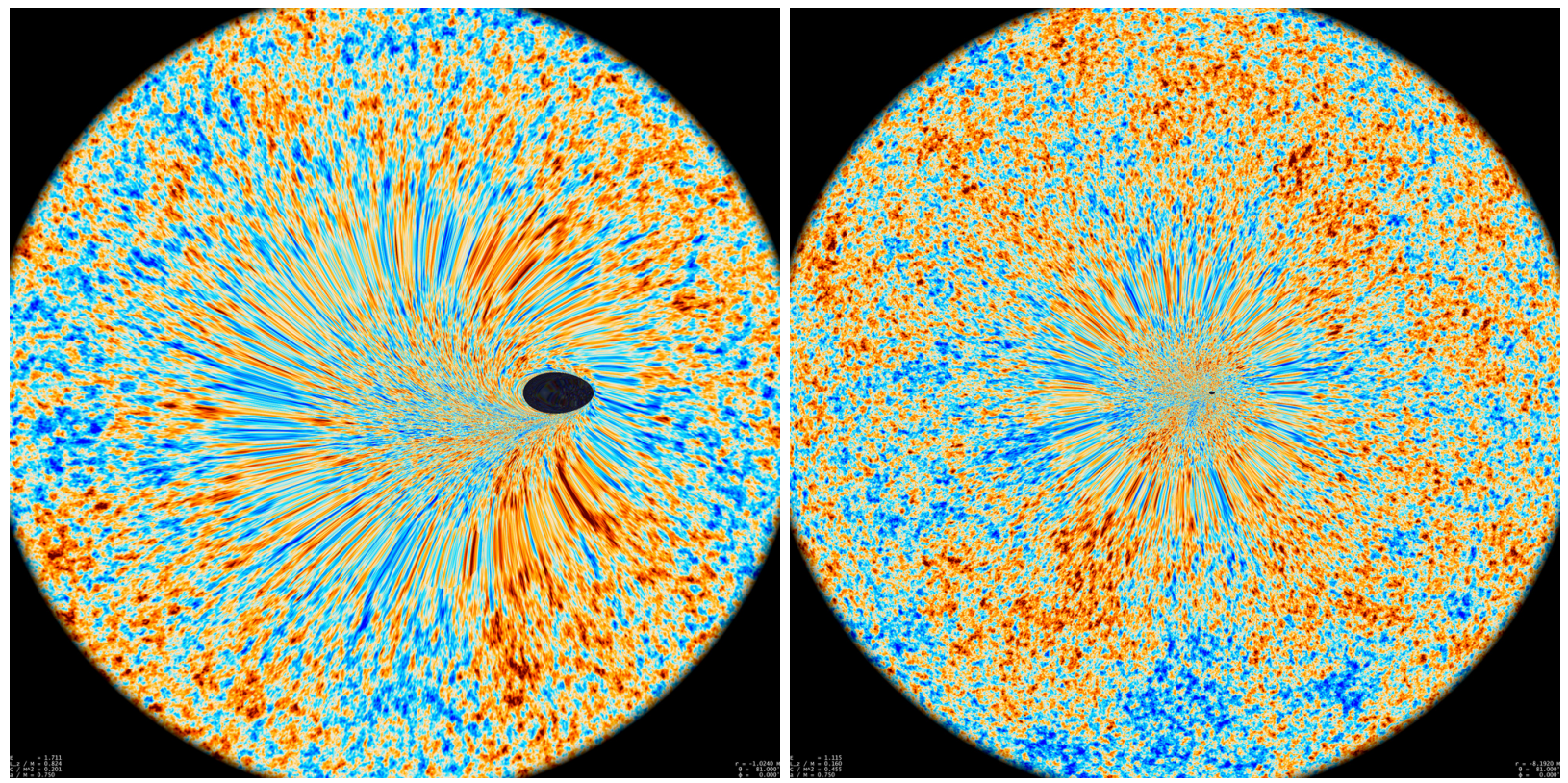

Fig. 19. Last steps of escape into the negative $r$ region, at $r=-1.0240 M$ and $-8.1920 M$.

itatively without using raytracing techniques, the variety of phenomena that arise is much larger than what can be expected. This study is far from being complete. We did not study the maximal analytic extension of the extremal Kerr wormhole nor the naked singularity, which we will devote to in a future work.

\section{Appendix A. Recovering the frame-dragging effect}

We show here that with the correct approximation, Eqns. 109110 lead to the well-known gravitomagnetic effect. The first step is to rewrite Eqns. (109 110) when neglecting $a$ as compared to $r$ since we consider a particle at (very) large distance from the black hole. In particular, this amounts to make the substitution $4 r^{2} / \Sigma-$ $3 \rightarrow 1$ and $4 r^{2} / \Sigma-1 \rightarrow 3$ and to neglect the $a \dot{\varphi}$ term as compared to $\dot{r}$. Moreover, we consider massive particles, so that $\kappa=1$. Overall, this gives

$$
\begin{aligned}
\ddot{x}+i \ddot{y}= & 4 i M a \frac{1}{r^{3}} W\left[\dot{x}+i \dot{y}-\frac{x+i y}{r}\left\{\dot{r}+\epsilon \frac{W^{2}-1}{4 W}\right\}\right] \\
& -M \frac{x+i y}{r^{3}}\left[1+3 \frac{C-a^{2} W^{2}}{r^{2}}\right], \\
\ddot{z}= & -M \frac{z}{r^{3}}\left[1+3 \frac{C}{r^{2}}\right] .
\end{aligned}
$$

The quantity $W$ is given in Eq. (31). Its physical meaning is the frequency shift of ingoing or outgoing radial null rays seen by the observer. At lowest order, it is 
easy to check that it is, as expected, equal to $1+\epsilon \dot{r}$. Therefore the term of the first equation above which is proportional to $\epsilon$ reduces to $\dot{r} / 2$. Finally, far from the black hole, and for a non relativistic observer $(\kappa=1, E \simeq 1)$, the Carter constant can be approximated as

$$
C \simeq \pi_{\theta}^{2}+a^{2}-2 a E L_{z}+\frac{L_{z}^{2}}{\mathrm{~s}^{2}} \simeq r^{4}\left(\dot{\theta}^{2}+\mathrm{s}^{2} \dot{\varphi}^{2}\right)+a^{2}-2 a L_{z} .
$$

The first two terms correspond to $L^{2}$, the observer's orbital angular momentum per unit of mass squared. Moreover, putting all the missing $c$ 's, the $a^{2}$ term is in fact $a^{2} c^{2}$ (still assuming that $a$ is a length, at most equal to the black hole coordinate radius). Consequently, $a^{2} c^{2}$ is not larger than $G^{2} \tilde{M}^{2} / c^{2}$, where here $\tilde{M}$ is the black hole mass in true units. In comparison, if we consider for simplicity an observer in orbit around the black hole at some distance $r$, the angular momentum per unit of mass is given by $r G \tilde{M}$. The ratio between the two quantities is therefore of order $L^{2} /\left(a^{2} c^{2}\right) \sim r c^{2} / G \tilde{M} \sim r / R_{\mathrm{BH}} \gg 1$. The $a^{2}$ term can therefore be dropped and we have

$$
\begin{aligned}
\ddot{x}+i \ddot{y}= & 4 i J \frac{1}{r^{3}}\left[\dot{x}+i \dot{y}-\frac{3}{2} \frac{\dot{r}}{r}(x+i y)\right] \\
& -\frac{x+i y}{r^{3}}\left[M+3 \frac{M L^{2}}{r^{2}}-6 \frac{J L_{z}}{r^{2}}\right], \\
\ddot{z}= & -\frac{z}{r^{3}}\left[M+3 \frac{M L^{2}}{r^{2}}-6 \frac{J L_{z}}{r^{2}}\right],
\end{aligned}
$$

Where $J=M a$ is the black hole spin. We recognize in these two equations the Newtonian term, proportional to $-M x^{i} / r^{3}$ plus the Schwarzschild term, proportional to $-M L^{2} x^{i} / r^{5}$. We need therefore to check that the first line of the first equation together with the $J L_{z}$ terms do corresponds to the usual gravitomagnetic term. If we express the metric in Cartesian Kerr-Schild coordinates, the $g_{t \tilde{\varphi}}$ term transforms into

$$
g_{t \varphi} \rightarrow g_{0 i}=\frac{2 J}{r^{3}}\left(\begin{array}{c}
-y \\
x \\
0
\end{array}\right) .
$$

The frame-dragging effect is recovered by computing the geodesic equation when considering only these terms and considering that the metric can be linearized with respect to this perturbation. For non relativistic trajectories, the only non-zero Christoffel symbols are $\Gamma_{i j}^{0}$ and $\Gamma_{0 j}^{i}, \Gamma_{j 0}^{i}$. If one is interested in the evolution of the Euclidean velocity vector $\boldsymbol{v}$, then the geodesic equation gives

$$
\dot{v}^{i}=-2 \Gamma_{0 j}^{i} v^{j},
$$

where the summation runs on the three spatial indices. The Christoffel symbols are given by

$$
\Gamma_{0 j}^{i}=-\Gamma_{i 0 j}=\frac{1}{2}\left(\partial_{i} g_{0 j}-\partial_{j} g_{0 i}\right)
$$


If we consider the three components of $g_{0 i}$ as those of a three-vector $\boldsymbol{g}$, then from Eq. A.6 we have

$$
\boldsymbol{g}=\frac{2 \boldsymbol{J} \wedge \boldsymbol{r}}{r^{3}}
$$

together with

$$
\dot{v}=\boldsymbol{v} \wedge(-\nabla \wedge \boldsymbol{g})
$$

The components of $-\nabla \wedge \boldsymbol{g}$ are

$$
-\nabla \wedge \boldsymbol{g}=\frac{2 J}{r^{5}}\left(\begin{array}{c}
-3 x z \\
-3 y z \\
r^{2}-3 z^{2}
\end{array}\right) .
$$

The components of the gravitational acceleration due to the frame-dragging are therefore

$$
\boldsymbol{a}=\frac{2 J}{r^{5}}\left(\begin{array}{c}
\dot{y} r^{2}-3 z^{2} \dot{y}+3 y z \dot{z} \\
-\dot{x} r^{2}+3 z^{2} \dot{x}-3 x z \dot{z} \\
3 z(x \dot{y}-y \dot{x})
\end{array}\right)
$$

The component along the $z$ axis can be rewritten using the fact that the constant of motion $L_{z}$ is equal to $x \dot{y}-y \dot{x}$, that is

$$
a_{z}=\frac{6 z J L_{z}}{r^{5}} .
$$

which is exactly the extra term of Eq. A.5. Regarding the $x$ and $y$ components, it is more convenient to use the complex combination $a_{x}+i a_{y}$ :

$$
a_{x}+i a_{y}=\frac{2 i J}{r^{5}}\left(\left(3 z^{2}-r^{2}\right)(\dot{x}+i \dot{y})-3 z \dot{z}(x+i y)\right) .
$$

In Eq. A.4 there are no $z$ nor $\dot{z}$, so that we now perform the substitution $z^{2} \rightarrow$ $r^{2}-x^{2}-y^{2}$ and $z \dot{z} \rightarrow r \dot{r}-x \dot{x}-y \dot{y}$, the last two terms of the last expression being re-expressed as

$$
x \dot{x}+y \dot{y}=(x-i y)(\dot{x}+i \dot{y})-i(x \dot{y}-y \dot{x})=(x-i y)(\dot{x}+i \dot{y})-i L_{z} .
$$

This leads to several simplifications whose net result is

$$
a_{x}+i a_{y}=i \frac{4 J}{r^{3}}\left(\dot{x}+i \dot{y}-\frac{3}{2} \frac{\dot{r}}{r}(x+i y)\right)+\frac{6 J}{r^{5}}(x+i y) L_{z},
$$

which corresponds to the extra terms of Eq. A.4. 


\section{Appendix B. Symmetrizing the observer in the inter-horizon region}

In this appendix, we compute the Lorenz factor that allows to go from the fourvelocity of an observer coming from region 1 with $E=1, L_{z}=0$ to its mirror analogue coming from region 3 with $E^{\prime}=-1, L_{z}^{\prime}=0$. This of course can only happen in region 2 (and 12 also). We assume that both of them have $\dot{\theta}=0$, so that they also have the same $\dot{r}$. The first observer's four-velocity has a norm of 1 , so that we can write, using the fact that $E=\pi_{t}$ and $L_{z}=-\pi_{\varphi}$,

$$
g^{t t} E^{2}-2 g^{t \varphi} E L_{z}+g^{\varphi \varphi} L_{z}^{2}+g_{r r} \dot{r}^{2}=1 .
$$

With our choice of constants of motion, this means that

$$
g_{r r} \dot{r}^{2}=1-g^{t t}
$$

The dot product between the two observers' four-velocity is then

$$
\gamma=g^{t t} E E^{\prime}-g^{t \varphi}\left(E L_{z}^{\prime}+E^{\prime} L_{z}\right)+g^{\varphi \varphi} L_{z} L_{z}^{\prime}+g_{r r} \dot{r}^{2} .
$$

This is of course the Lorentz factor of the boost that transforms one four-velocity into the other. Again, all the $L_{z}, L_{z}^{\prime}$ terms cancel away and we have

$$
\gamma=1-2 g^{t t}=1+2 \frac{1}{(-\Delta)}\left(r^{2}+a^{2}+\frac{2 M r a^{2} s^{2}}{\Sigma}\right) .
$$

Given the observer's position we are considering $\left(\theta=81^{\circ}, r=M\right.$, the Lorentz factor is close to 9 , which corresponds to a fairly large boost indeed. Even going from one observer to the "lazy", intermediate, observer with $E=0$ gives a Lorentz factor of $\simeq 2.23$, that is, a relative velocity close to $0.9 \mathrm{c}$. For other values of $r$, the Lorentz factor are even larger (and diverge as $r$ gets close to any of the two horizons, see also ${ }^{21}$ for the Reissner Nordström case).

\section{References}

1. R.P. Kerr, in I. Robinson, A. Schild \& E.L. Schucking (eds.), 1st Texas Symposium on Relativistic Astrophysics, University of Chicago Press, Chicago, United States (1965), pp. 99-102.

2. R.H. Boyer \& T.G. Price, Mathematical Proceedings of the Cambridge Philosophical Society, 61, 531-534 (1965).

3. S. U. Viergutz, Astron. Astrophys., 272, 355 (1993).

4. C. Fanton et al., Publ. Astr. Soc. Jap., 49, 159-169 (1997).

5. H. Falcke, F. Melia \& E. Agol, Astrophysical Journal Letters, 528, L13-L16 (2000), arXiv:9912263 (astro-ph).

6. K. Beckwith \& C. Done, Month. Not. Roy. Astr. Soc., 359, 1217-1228 (2005), arXiv:0904.3575 (astro-ph).

7. O. James et al., Class. Quant. Grav., 32, 065001 (2015), arXiv:1502.03808 (gr-qc).

8. M.S. Morris \& K.S. Thorne, American Journal of Physics, 56, 395-412 (1988).

9. R. Penrose, in Battelle Rencontres: $196^{7}$ Lectures in Mathematics and Physics, C. de Witt-Morrette \& J. A. Wheeler Eds, 112-235 (1968). 
10. M. Visser, in D. Wiltshire, M. Visser \& S. Scott (eds.), The Kerr spacetime: Rotating Black Holes in General Relativity, Cambridge, United Kingdom (2009), pp. 3-37, arXiv:0706.0622 3 (gr-qc).

11. S.A. Teukolsky, Class. Quantum Grav., 32, 124006 (2015), arXiv:1410.2130v2 (gr-qc).

12. S. Chandrasekhar, The Mathematical Theory of Black Holes, Oxford University Press, Oxford, England (1983).

13. B. O'Neill, The Geometry of Kerr Black Holes, AK Peters, Ltd., Wellesley, Massachusetts, United States (1995).

14. A. Riazuelo, Int. Journ. Mod. Phys. D, 28, 1950042 (2019), arXiv:1511.06025 (gr-qc).

15. M.F. Skrutskie, et al., The Astronomical Journal, 131, 1163-1183. (2006). The pixellized celestial sphere that we used is at URL https://old.ipac.caltech.edu/2mass/gallery/showcase/allsky/fullres.html .

16. R.H. Boyer \& R.W. Lindquist, Journal of Mathematical Physics, 8, 265-281 (1967).

17. S.W. Hawking \& G.F.R. Ellis, The large-scale structure of space-time, Cambridge University Press, Cambridge, England (1973).

18. B. Carter, Phys. Rev., 174, 1559-1572 (1968).

19. J.-A. Marck, Class. Quant. Grav., 13, 393-402 (1996), arXiv:9505010 (gr-qc).

20. E. Hackmann, Geodesic equations in black hole space-times with cosmological constant, Bremen University dissertation (2010) http://elib.suub.unibremen.de/diss/docs/00011880.pdf

21. A. Riazuelo, Int. Journ. Mod. Phys. D, 28, 1950084 (2019), arXiv:1812.07705 (gr-qc).

22. W. H. Press et al., Numerical Recipes in $C, 2^{\text {nd }}$ edition, Cambridge University Press, Cambridge, Great Britain (1992).

23. P.A.R. Ade, et al., Astronomy \& Astrophysics, 536, A1 (2011), arXiv:1101.2022 (astro-ph). High resolution image in equirectangular projection available at URL http://astrog80.astro.cf.ac.uk/Planck/Planckoscope-tiles/fullsky/ .

24. R. Adam, et al., Astronomy \& Astrophysics, 594, A1 (2016), arXiv:1502.01582 (astroph). Image available at URL http://planck.cf.ac.uk/all-sky-images/

25. C.L. Bennett, et al., The Astrophysical Journal Supplement Series, 208, 20 (2013), arXiv:1212.5225 (astro-ph). Image available at URL https://lambda.gsfc.nasa.gov/product/map/current/m_images.cfm .

26. R. Penrose, Mathematical Proceedings of the Cambridge Philosophical Society, 55, 137-139 (1959). 